\title{
Survival of Hedge Funds: Frailty vs Contagion*
}

\author{
Serge Darolles ${ }^{\dagger}$, Patrick Gagliardini ${ }^{\ddagger}$, Christian Gouriéroux ${ }^{\S}$
}

First version: April 2011

This version: November 2012

${ }^{*}$ Acknowledgements: We thank T. Adrian, P. Christoffersen, J. Jasiak, I. Makarov, C. Meghir, A. Melino, B. Schwaab and participants at the Computational and Financial Econometrics Conference 2011 in London, the Third Annual Conference on Hedge Funds 2011 in Paris, the International Conference on Stochastic Analysis and Applications 2011 in Hammamet, the ESEM 2012 in Malaga, the Conference on Recent Developments in Econometrics 2012 in Toulouse and seminars at Toronto University and ESSEC Business School for useful comments. We gratefully acknowledge financial support of the chair QUANTVALLEY/Risk Foundation: "Quantitative Management Initiative” and the Swiss National Science Foundation through the NCCR FINRISK network.

†Paris Dauphine University and CREST.

$\ddagger$ University of Lugano and Swiss Finance Institute.

${ }^{\S}$ University of Toronto and CREST. 


\title{
Survival of Hedge Funds: Frailty vs Contagion
}

\begin{abstract}
In this paper we examine the dependence between the liquidation risks of individual hedge funds. This dependence can result either from common exogenous shocks (shared frailty), or from contagion phenomena, which occur when an endogenous behaviour of a fund manager impacts the Net Asset Values of other funds. We introduce dynamic models able to distinguish between frailty and contagion phenomena, and test for the presence of such dependence effects, according to the age and management style of the fund. We demonstrate the empirical relevance of our approach by measuring the magnitudes of contagion and exogenous frailty in liquidation risk dependence in the TASS database. The empirical analysis is completed by stress-tests on portfolios of hedge funds.

Keywords: Hedge Fund, Liquidation Correlation, Frailty, Contagion, Dynamic Count Model, Autoregressive Gamma Process, Systemic Risk, Stress-tests, Liquidation Swap, Funding Liquidity, Market Liquidity.
\end{abstract}

JEL classification: G12, C23. 


\section{Introduction}

The rather short lifetimes ${ }^{1}$ of a majority of hedge funds (HF) and the reasons of the dependence between their liquidations explain the interest of investors, academics and regulators in HF survival analysis. There exist different economic reasons explaining why a HF manager decides to liquidate a fund. Loosely speaking, this decision can be taken since either the income of the fund manager is not sufficient, or the market conditions are not appropriate for managing the fund in an efficient way. Let us discuss these two aspects.

\section{i) Large cash withdrawals}

The income of a fund manager is coming from management fees which are indexed in a complicated way on the performance of the fund, but also on the total Asset Under Management (AUM). In particular, when outflows are important it may become uninteresting to continue to manage the fund. Moreover, this effect is amplified by the specific high water mark fee structure implemented by the HF manager [see e.g. Brown, Goetzmann, Liang (2004), Darolles, Gourieroux (2012) for the description of fees]. This is the so-called L-effect, L for liability, since it impacts the liability component of the balance sheet of the fund. This L-effect can be a source of liquidation dependence, for instance during a funding liquidity crisis when the fund managers experience jointly large cash withdrawals, while having difficulty in obtaining credit and being obliged to diminish their use of leverage. This effect also arises with the withdrawal of some prime brokers, and it is amplified by the use of debt to create the needed leverage. If the prime brokers simultaneously quit several funds, we get a frailty phenomenon, that is, a common risk factor. This frailty effect is exogenous, even when there is a herd-

\footnotetext{
${ }^{1}$ The global annual liquidation rate for hedge funds between 1994 and 2003 has been around 8\%-9\%, which corresponds to a median lifetime of 6-7 years. However, the liquidation rate is considerably varying according to the year and management style, with values between about $4 \%$ and $30 \%$ for that period [see e.g. Getmanski, Lo, Mei (2004), Chan, Getmanski, Haas, Lo (2007), Table 6.14]. Moreover, the liquidation rate depends significantly on the definition of liquidation and on the database.
} 
ing behaviour of prime brokers, as along as their decision is not triggered by past liquidation events.

\section{ii) Market effect}

If $\mathrm{HF}$ portfolios are invested in illiquid assets, it can be difficult and risky to continue to manage funds during a market liquidity crisis. Indeed, the fire sales of a given fund manager will consume the market liquidity of a given class of illiquid assets. The first consequence of such fire sales is a price pressure on these assets, which implies a decrease of the market value of all funds holding these assets in their portfolio. This is the A-effect, A for assets, since it concerns the asset component of the balance sheet. This effect is often called contagion in the HF literature. A well-known example is the default of the Russian sovereign debt in August 1998, when Long Term Capital Management (LTCM) and many other fixed-income HF suffered catastrophic losses over the course of a few weeks. Then, the failure of one of these funds increases the probability of liquidation of other funds.

iii) Finally, liquidation can also be the consequence of illegal operations, such as fraud, unauthorized trading and misappropriation of investors funds, of conflicts of interest or inadequate resources. The analysis of operational risk is out of the scope of our paper and it is expected that the new regulations will diminish the importance of this reason for HF liquidation. ${ }^{2}$

From the investor's point of view, liquidation is a risk, which concerns both the timing of the payoffs and the value of the fund at liquidation time. It is partially at the discretion of the fund manager, who can slow down or accelerate liquidation by an appropriate management of gates, for instance. The liquidation risk is similar to default risk, prepayment risk, or lapse risk encountered on

\footnotetext{
${ }^{2}$ At the beginning of the 2000's, about half of all failures might be attributed to such operational risks [Feffer, Kundro (2003)]. On October 2, 2004, the SEC required the managers of the main hedge funds to register as investment advisors by February 1, 2006. Such request applies to the hedge funds whose manager is based in the United States, with more than 14 clients, assets of at least 25 millions USD and a lockup period of less than two years. The managers have to fill the Form ADV and reveal the operational risk problems encountered in the past. Among the registered individual funds alive in 2006 , about $16 \%$ encountered operational difficulties in the past [Brown et al. (2008)].
} 
corporate bonds, credit derivatives, or life insurance contracts, respectively. Liquidation risk is often neglected in standard portfolio management practices. It is a hidden risk, in contrast to the visible risk directly measured by the volatility of returns, i.e. the market risk. A fund with a small visible market risk can have a large hidden liquidation risk. Liquidation risk is especially important for the following three types of market participants:

i) Most of the money invested in HF in the yearly 2000's comes from institutional investors, including endowments, foundations, corporate and public plans, and insurance (if they have a minimal capital) [Casey, Quirk, Acito (2004)]. They invest on a long term basis, are interested in the low correlation of HF returns with traditional assets classes, like equity and bonds, and want to avoid the consequences of a short term liquidity crisis.

ii) The funds of funds can be very sensitive to liquidation risk dependencies between individual funds. An appropriate survival analysis can help to detect the funds of funds, which are too sensitive to such systematic risk.

iii) The regulators have to monitor both the L- and A- effects. They could want to modify and control the funding liquidity exposure by means of restrictions on the use of leverage, of the redemption frequency and of the minimal requirements for investing in a HF. Regulators could also want to limit the market liquidity exposure by applying the Basel III approach, for instance by introducing additional reserves based on liquidity stress scenarios.

In this paper we focus on the liquidation times and do not consider the losses on Net Asset Value during the liquidation process. ${ }^{3}$ The analysis of HF lifetimes is generally based on a duration model,

\footnotetext{
${ }^{3}$ It would be much more difficult to measure the loss given liquidation of hedge funds than the loss given default of large corporations for three reasons. i) The exposure at liquidation are self-reported and have to be carefully checked, ii) The hedge funds cannot issue bonds for refinancing and thus there exists no market value of their liquidation risk, iii) The hedge fund portfolios can include a significant proportion of illiquid assets, which require time to be sold at a reasobale price during the liquidation process.
} 
which can be either parametric, semi-parametric, or nonparametric. Typically, in a first step analysis researchers study how the liquidation intensity depends on the age of the HF, and/or on calendar time. This is done by appropriately averaging the observed liquidation rates [see e.g. the Kaplan-Meier non parametric estimation of the hazard function in Baba, Goko (2006), Figure 1, for the description of age dependence, or the log-normal hazard function used in Malkiel, Saha (2005)]. In a second step, a parametric specification of the (discrete-time) liquidation intensity can be selected, such as a logit, or a probit model to analyze the possible determinants of liquidation. The explanatory variables can be time independent HF characteristics, such as the management style, the domicile country (offshore vs domestic), the minimum investment, variables summarizing the governance structure, such as the existence of incentive management fees, and their design (high water mark, hurdle rate), the announced cancellation policy (redemption frequency, lockup period), the experience and education level of the manager [Boyson (2010)]. Regressors can also include time dependent HF individual characteristics, such as lagged individual HF return, realized return volatility and skewness, asset under management (AUM) and the recent fund inflows [see e.g. Baquero, Horst, Verbeek (2005), Malkiel, Saha (2005), Chan, Getmansky, Haas, Lo (2007), Section 6.5.1, Boyson (2010)], as well as time dependent market characteristics [Chan, Getmansky, Haas, Lo (2007), Section 6.6.1, Carlson, Steinman (2008)] and the competitive pressure, measured by the total number of HFs [Getmansky (2010)]. Finally, the parametric and nonparametric approaches can be combined in the proportional hazard model introduced by Cox (1972), as in Brown, Goetzmann, Park (2001), Baba, Goko (2006), Gregoriou, Lhabitant, Rouah (2010).

While the above survival models are useful for a descriptive analysis of liquidation intensity, these models are not always appropriate for liquidation risk prediction, for evaluation of systematic risk, or for capturing the observed liquidation clustering, as required in a stress testing analysis. For instance, for such purposes it is not suitable to introduce time dependent explanatory variables in survival mod- 
els. Indeed, the future liquidation risk can only be analyzed after predicting the future values of the time dependent explanatory variables. This is a difficult task as it requires a joint dynamic model for these variables and the liquidation indicators. Furthermore, since the duration models considered in the literature assume the independence of individual liquidation risks given the selected observed explanatory variables, liquidation correlation has not yet been included in the HF survival models.

Based on the above discussion, two causes of liquidation risk dependencies arise.

i) There exist underlying exogenous stochastic factors, which have a common influence on the liquidation intensities of the individual HF. In the credit risk literature, these factors are called systematic risks, or frailties [Duffie et al. (2009)]. Such common exogenous shocks also exist in the joint analysis of HF lifetimes and capture the funding liquidity risk, that is, the L-effect.

ii) Risk dependency can also arise when a shock to one fund has an impact on other funds. This is the so-called contagion effect. In the case of credit risk, contagion is generally due to the debt structure, when some banks or funds invest in other banks or funds. For HFs it corresponds to the market liquidity risk, that is, the A-effect.

The aim of our paper is to introduce both frailty and contagion effects in HF survival models, and to measure the magnitudes of these effects. The liquidation intensity of an individual HF is assumed to depend on the lagged observations of liquidation counts in the same and in the other management styles, as well as on a common unobservable dynamic factor. The former explanatory variables represent contagion (A-effect), the latter represents the shared frailty (L-effect). The specification allows to disentangle the two types of liquidation risk dependence by exploiting the time lag that contagion necessitates to produce its effects. Such a model is especially appropriate to analyze in a dynamic framework the consequences of stress on either funding or market liquidity, and then to design the possible policies to diminish some of these consequences. The analysis of underlying common risk factors and contagion is the first step before studying the impact of HF on systemic risk for the global 
financial markets, and the possible cascade into a global financial crisis.

The paper is organized as follows. In Section 2 we first introduce the microscopic foundation for our framework based on individual hedge funds. A macroscopic Poisson model with both common frailty and contagion is derived by aggregating liquidation counts by management styles and age classes. The Poisson model with contagion and autoregressive gamma frailty is especially convenient, since it provides a joint affine dynamics for the frailty and the liquidation counts. This facilitates the prediction of future liquidation risk as well as the estimation of parameters in such a nonlinear setting with unobservable factors. Dynamic models with contagion and frailty are estimated on HF data in Section 3. We assess the relative magnitude of contagion and shared frailty phenomena when we study liquidation risks dependence across different management styles. We carefully distinguish between the direct frailty effect and the amplification of the exogenous systematic shocks through the contagion network. We illustrate our methodology by an application to dynamic stress tests of HF portfolios, that evaluates the stress effects on the term structure of liquidation risk. Section 4 concludes. Technical proofs are gathered in appendices and supplementary materials.

\section{Frailty vs Contagion in liquidation of hedge funds by genera-}

\section{tion and type}

In this section we introduce a dynamic model for the joint distribution of liquidation count histories of hedge funds of different types. The cross-sectional dependence between individual HF liquidation risks, and the liquidation risk dynamics, are captured by introducing $i$ ) the autoregressive effect of lagged counts, that is the contagion effect, and ii) a dynamic frailty to represent unobservable exogenous common shocks. 


\subsection{Microfoundations}

Let us partition the set of HF according to a qualitative individual characteristic, called the type. The type depends on the hedge fund, and is time independent. This individual characteristic can be the management style, the domicile state/country, the minimum investment, the type of governance, or the crossing of such variables. The type can take $K$ alternatives, $k=1, \ldots, K$. Moreover, let $h$ denote the age of the fund, where $h=1, \ldots, H$.

For each period $t$, we denote by $n_{k, h, t}$ the number of hedge funds with age $h$ and type $k$ at the beginning of the period. Among these $n_{k, h, t}$ hedge funds, $Y_{k, h, t}$ will be liquidated during this period. The parametric model specifies the joint distribution of the number of liquidations $Y_{k, h, t}$, for $k, h$ and $t$ varying. We first define the joint distribution of these liquidation counts given the common frailty history. Then, the model is completed by specifying the frailty dynamics, which is needed to account for the unobservability of the common stochastic factors.

Let us denote by $F_{t}$ the factor value at date $t$, and by $Y_{t}=\left(Y_{k, h, t}, k=1, \ldots, K, h=1, \ldots, H\right)^{\prime}$ the vector of liquidation counts in period $t$ for all fund types and ages. Let $\underline{F_{t}}=\left(F_{t}, F_{t-1}, \ldots\right)$ and $\underline{Y_{t}}=\left(Y_{t}, Y_{t-1}, \ldots\right)$ denote the factor and liquidation counts histories, respectively, at date $t$. The factor $F_{t}$ can be multidimensional with dimension $m$. We make the following assumption:

Assumption A.1: Conditional on $\underline{F_{t}}$ and $\underline{Y_{t-1}}$, the liquidation counts $Y_{k, h, t}$, for $k, h$ varying, are independent with binomial distribution:

$$
Y_{k, h, t} \sim \mathcal{B}\left[n_{k, h, t}, p_{k, h, t}(\theta)\right]
$$

where $p_{k, h, t}(\theta)$ denotes the discrete-time liquidation stochastic intensity in period t for category $(k, h)$, and $\theta$ is an unknown parameter.

The liquidation intensity is time varying and stochastic, since it depends on the unobservable stochastic frailty process $\left(F_{t}\right)$ and on the lagged liquidation counts. In general we consider transfor- 
mations of the liquidation rates by the inverse of a cumulative distribution function (c.d.f.) to ensure $p_{k, h, t}(\theta)$ to be between 0 and 1 . We get a logit (resp. probit) model when the c.d.f. corresponds to the logistic distribution (resp. standard Gaussian distribution). We consider below the c.d.f. of the exponential distribution since this transformation provides the continuously compounded liquidation intensity, ${ }^{4}$ which is specified as:

$$
-\log \left[1-p_{k, h, t}(\theta)\right]=a_{k, h}(\theta)+b_{k, h}(\theta)^{\prime} F_{t}+c_{k, h}(\theta)^{\prime} Y_{t-1}^{*},
$$

where vector $Y_{t-1}^{*}=\left(Y_{1,1, t-1}^{*}, \ldots, Y_{1, H, t-1}^{*}, \ldots, Y_{K, 1, t-1}^{*}, \ldots, Y_{K, H, t-1}^{*}\right)^{\prime}$ measures the lagged liquidation rates at the source of possible contagions. The common factor and the parameters are assumed such that the RHS of equation (2.2) is positive. The specification of the liquidation rate includes marginal effects of the individual type and age by means of $a_{k, h}(\theta)$, cross effects of type and age with frailty through $b_{k, h}(\theta)^{\prime} F_{t}$, and cross effects of type and age with lagged counts. The variable $Y_{k, h, t-1}^{*}$ can be chosen as the lagged liquidation count $Y_{k, h, t-1}$, or lagged liquidation frequency $Y_{k, h, t-1} / n_{k, h, t-1}$, according to the degree of integration of the HF market. Thus, an effect of the lagged class size can be represented by the size adjusted variables $Y_{t-1}^{*}$. The current size variable $n_{k, h, t}$ could also be introduced in the liquidation intensity as the third type of explanatory variable to capture a competitive pressure effect. Loosely speaking, if $n_{k, h, t}$ is small, the default of one HF can improve the monopolistic power of the surviving HF's in this category and thus diminish their future liquidation rates. The contagion effects can be both within and between categories. The vector of sensitivity coefficients $c_{k, h}(\theta)$ has components $c_{k, h, k^{\prime}, h^{\prime}}(\theta)$, for $k^{\prime}, h^{\prime}$ varying, which measure the magnitude of contagion from category $\left(k^{\prime}, h^{\prime}\right)$ to category $(k, h)$. This contagion effect is not necessarily symmetric. For example, we can have contagion from $\left(k^{\prime}, h^{\prime}\right)$ to $(k, h)$ without contagion from $(k, h)$ to $\left(k^{\prime}, h^{\prime}\right)$.

The frailty dynamics in specified in the following assumption:

Assumption A.2: The shared frailty process $\left(F_{t}\right)$ is unobservable. It is an exogenous Markov process

\footnotetext{
${ }^{4}$ that is, an affine specification of the stochastic intensity in continuous time modeling [see equation (2.9)].
} 
with transition density:

$$
g\left(f_{t} \mid f_{t-1} ; \varphi\right)
$$

where $\varphi$ is an unknown parameter.

Under Assumption A.2, the transition of factor process $\left(F_{t}\right)$ depends on the lagged factor value only, and it does not depend on the lagged liquidation counts $\underline{Y_{t-1}}$. Thus, the factor dynamic is exogenous and $F_{t}$ summarizes the exogenous shocks affecting jointly the liquidation intensities of the different categories $(k, h)$ at the beginning of period $t$. As discussed in the Introduction, the most relevant economic interpretation of the common factor is likely a measure of funding liquidity risk.

The notion of frailty has been initially introduced in duration models in Vaupel, Manton, Stallard (1979) and later used to define the Archimedean copulas [Oakes (1989)]. In this meaning, the frailty is an unobservable individual variable introduced to account for omitted individual characteristics and correct for the so-called mover-stayer phenomenon [see e.g. Baba, Goko (2006) for the introduction of an individual static frailty in the $\mathrm{HF}$ literature]. In our framework, $F_{t}$ is indexed by time and common to all HF, which justifies the terminology "dynamic frailty" introduced by Duffie et al. (2009) for application to credit risk. The unobservable common frailty (also called systematic risk) has to be integrated out, which creates a contemporaneous dependence between the individual HF liquidation risks as well as long memory features. The coefficient $b_{k, h}(\theta)$ in equation (2.2) gives the sensitivity of the continuously compounded liquidation rate of category $(k, h)$ with respect to this shared frailty. In general, this coefficient depends on both fund type $k$ and age $h$. For instance, we expect that the newly created HFs are more robust, and thus less sensitive to exogenous shocks during the first 2 years, when they have survivorship available reserves. Then the liquidation rates will increase between 2 and 4 years to diminish later on when the HF becomes mature. However, it is not yet known how the liquidation correlation depends on the age, and how these different age effects vary with the HF type.

The serial and cross-sectional correlation between liquidation counts can be due to either shared 
frailty (systematic risk), or contagion. This explains why it is important to test for the existence of such effects. In particular, specification (2.1)-(2.2) includes as special cases:

i) the model without frailty and contagion, if $b_{k, h}(\theta)=0$ and $c_{k, h}(\theta)=0, \forall k, h$.

ii) The model with frailty only, when $c_{k, h}(\theta)=0, \forall k, h$.

iii) The model with contagion only, when $b_{k, h}(\theta)=0, \forall k, h$. This submodel is a time discretized version of the self- and mutually-exciting point processes introduced by Hawkes (1971) and Hawkes, Oakes (1974). Models of this type are used in the analysis of corporate default clustering by Giesecke and Weber (2004), and for studying contagion across financial markets by Ait-Sahalia, Cacho-Diaz, Laeven (2010); see also Dungey et al. (2010) for a review on contagion models.

iv) The proportional hazard model with both frailty and contagion, if $a_{k, h}(\theta)=d_{h}(\theta) a_{k}^{0}(\theta), b_{k, h}(\theta)=$ $d_{h}(\theta) b_{k}^{0}(\theta)$ and $c_{k, h}(\theta)=d_{h}(\theta) c_{k}^{0}(\theta)$, say. Then, $d_{h}(\theta)$ is the baseline liquidation intensity, defined up to a multiplicative scale.

Frailty and contagion both create correlation between the lifetimes of individual HF. In the limiting case of a static model, that is, with a serially independent frailty process $\left(F_{t}\right)$ and simultaneous effects of observed liquidation counts, the two phenomena cannot be identified. This is the reflection problem highlighted in Manski (1993). In the dynamic framework of Assumptions A.1 and A.2, frailty and contagion can be disentangled, since the frailty has a contemporaneous effect while contagion is produced only after a time lag [see Gagliardini and Gourieroux (2012) for a discussion of identification in models with latent dynamic factors]. Our paper is similar in spirit to Azizpour, Giesecke (2008), that considers a model for corporate default intensity including a common factor and a self-exciting jump component. However, the model in Azizpour, Giesecke (2008) is written at the aggregate level of the economy, and allows neither for distinguishing within and between group contagion, nor for heterogeneity in the sensitivity coefficients to the shared frailty.

Let us now derive the likelihood function of the binomial model in Assumptions A.1 and A.2. 
Conditional on the factor history, the joint density of the liquidation counts of all management styles and age categories is:

$$
\prod_{k=1}^{K} \prod_{h=1}^{H} \prod_{t=1}^{T}\left\{\left(\begin{array}{c}
n_{k, h, t} \\
y_{k, h, t}
\end{array}\right)\left[p_{k, h, t}(\theta)\right]^{y_{k, h, t}}\left[1-p_{k, h, t}(\theta)\right]^{n_{k, h, t}-y_{k, h, t}}\right\}
$$

Since the common dynamic frailty is unobservable, the likelihood function is deduced by integrating out the factor history. Thus, the likelihood function is given by:

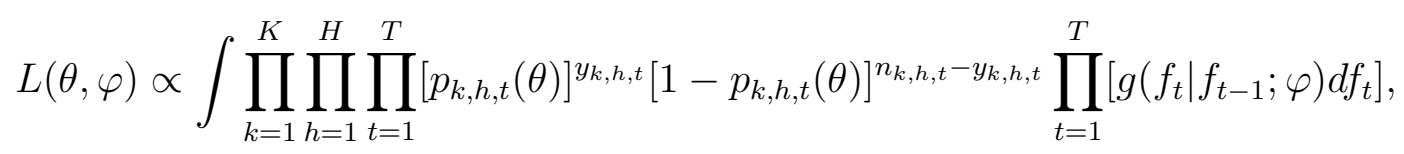

where $\propto$ means equality up to a scale factor independent of parameters.

The likelihood function in (2.5) has a complicated expression, which involves a $m T$-dimensional integral, whenever a shared dynamic frailty is introduced in the model. Despite the complicated likelihood function, the model is suitable for simulating factor and liquidation counts histories, and can be estimated by the simulation based methods. Indeed, this model is a nonlinear state space model, and the Gibbs sampling can be used to evaluate numerically the likelihood function [see e.g. Cappé, Moulines, Rydén (2005), and Duffie et al. (2009) for an application in credit risk]. However, the Gibbs sampling can be time consuming and difficult to implement numerically, especially when there is a large number of parameters to estimate. For this reason, we consider next a Poisson approximation of the binomial model, and develop in Section 3 a new method of moments for its estimation.

\subsection{The macroscopic model}

When the sizes of the categories are large, the binomial model in Assumption A.1 can be approximated by the Poisson model with both contagion and frailty. The Poisson model is very convenient, as it provides a joint affine dynamics for the frailty and the liquidation counts. This facilitates the filtering of the unobservable factor, the nonlinear prediction of future liquidation risk as well as the estimation of the parameters. 
The HF categories are large and the liquidation rates are small, when monthly data are used. Then, the binomial distribution can be well-approximated by the Poisson distribution [see e.g. Czado, Delwarde, Denuit (2005) and Gagliardini, Gourieroux (2011) for such an approximation in life insurance contracts and credit risk analysis, respectively]. To derive such an approximation let us assume that the category sizes are such that:

$$
n_{k, h, t} \simeq \gamma_{k, h, t} n
$$

where $n$ tends to infinity and $\gamma_{k, h, t}$ are fixed coefficients, that are, independent of $n$. Let us also assume that the liquidation intensities are such that:

$$
\lim _{n \rightarrow \infty} n p_{k, h, t}(\theta)=\lambda_{k, h, t}(\theta) \geq 0, \text { say. }
$$

Then, the conditional distribution of the liquidation counts can be approximated by a Poisson distribution:

$$
Y_{k, h, t} \sim \mathcal{P}\left[\gamma_{k, h, t} \lambda_{k, h, t}(\theta)\right]
$$

When model (2.2) is chosen for the liquidation intensity, its expansion provides an affine form for the limiting liquidation intensity:

$$
\lambda_{k, h, t}(\theta)=a_{k, h}(\theta)+b_{k, h}(\theta)^{\prime} F_{t}+c_{k, h}(\theta)^{\prime} Y_{t-1}^{*}, \text { say }
$$

The scale component $\gamma_{k, h, t}$ in (2.6) is a size adjustment, which can be replaced for instance by:

$$
\hat{\gamma}_{k, h, t}=n_{k, h, t} / n_{k, h, t_{0}}
$$

where $t_{0}$ is a given date. This adjustment is especially useful for the HF industry, which has experienced a large development before the crisis of 2008 (see Section 3.2). Due to this size adjustment, the distribution of the liquidation count $Y_{k, h, t}$ depends on the current sample size $n_{k, h, t}$.

While equation (2.1) defines the model for a finite population, equation (2.8) defines its limit for a large population size. Below we show that, by disregarding the effect of finite category size, 
we simplify the dynamics and obtain an affine dynamic for the joint process of liquidation counts and frailty [see e.g. Duffie, Filipovic, Schachermayer (2003) for continuous time affine processes, and Darolles, Gourieroux, Jasiak (2006) for discrete time]. The Poisson approximation leads to the conditional Laplace transform of the current liquidation counts vector $Y_{t}$ given $\underline{F_{t}}$ and $\underline{Y_{t-1}}$, which is:

$$
\begin{aligned}
\psi_{t}(u)= & E\left[\exp \left(-u^{\prime} Y_{t}\right) \mid \underline{F_{t}}, \underline{Y_{t-1}}\right]=\prod_{k, h} E\left[\exp \left(-u_{k, h} Y_{k, h, t}\right) \underline{F_{t}}, \underline{Y_{t-1}}\right] \\
= & \prod_{k, h} \exp \left\{-\gamma_{k, h, t} \lambda_{k, h, t}(\theta)\left[1-\exp \left(-u_{k, h}\right)\right]\right\} \\
= & \exp \left\{-\sum_{k, h}\left[1-\exp \left(-u_{k, h}\right)\right] \gamma_{k, h, t} a_{k, h}(\theta)-\sum_{k, h}\left[1-\exp \left(-u_{k, h}\right)\right] \gamma_{k, h, t} b_{k, h}(\theta)^{\prime} F_{t}\right. \\
& \left.-\sum_{k, h}\left[1-\exp \left(-u_{k, h}\right)\right] \gamma_{k, h, t} c_{k, h}(\theta)^{\prime} Y_{t-1}^{*}\right\},
\end{aligned}
$$

where $u$ is a vector with nonnegative components $u_{k, h}$.

The conditional Laplace transform (2.11) is an exponential affine function of $F_{t}$ and $Y_{t-1}^{*}$. By selecting an affine dynamics for the latent factor process $\left(F_{t}\right)$, that is, by assuming that:

$$
E\left[\exp \left(-u^{\prime} F_{t}\right) \mid \underline{F_{t-1}}, \underline{Y_{t-1}}\right]=\exp \left[-\alpha(u)^{\prime} F_{t-1}-\beta(u)\right]
$$

for some positive functions $\alpha$ and $\beta$, we deduce also an affine dynamic for the joint process $\left(Y_{t}^{\prime}, F_{t}^{\prime}\right)^{\prime}$. Specifically, the joint conditional Laplace transform of process $\left(Y_{t}, F_{t}\right)$ is deduced from (2.11)-(2.12) by iterated expectation, and we get:

$$
\begin{aligned}
\psi_{t}(u, v)= & E\left[\exp \left(-u^{\prime} Y_{t}-v^{\prime} F_{t}\right) \underline{F_{t-1}}, \underline{Y_{t-1}}\right] \\
= & E\left\{E\left[\exp \left(-u^{\prime} Y_{t}\right) \underline{F_{t}}, \underline{Y_{t-1}}\right] \exp \left(-v^{\prime} F_{t}\right) \mid \underline{F_{t-1}}, \underline{Y_{t-1}}\right\} \\
= & \exp \left\{-\sum_{k, h} \gamma_{k, h, t} a_{k, h}(\theta)\left[1-\exp \left(-u_{k, h}\right)\right]-\sum_{k, h}\left[1-\exp \left(-u_{k, h}\right)\right] \gamma_{k, h, t} c_{k, h}(\theta)^{\prime} Y_{t-1}^{*}\right. \\
& -\alpha\left(\sum_{k, h} \gamma_{k, h, t} b_{k, h}(\theta)\left[1-\exp \left(-u_{k, h}\right)\right]+v\right)^{\prime} F_{t-1} \\
& \left.-\beta\left(\sum_{k, h} \gamma_{k, h, t} b_{k, h}(\theta)\left[1-\exp \left(-u_{k, h}\right)\right]+v\right)\right\}
\end{aligned}
$$


This joint conditional Laplace transform is exponential affine in lagged values $Y_{t-1}$ and $F_{t-1}$, that is, it is of the form:

$$
\psi_{t}(u, v)=\exp \left\{-\alpha_{1, t}(u, v)^{\prime} Y_{t-1}-\alpha_{2, t}(u, v)^{\prime} F_{t-1}-\beta_{t}(u, v)\right\}, \text { say, }
$$

where the sensitivity coefficients $\alpha_{1, t}, \alpha_{2, t}$ and $\beta_{t}$ depend on the class size and are time dependent in general. Thus, process $\left(Y_{t}^{\prime}, F_{t}^{\prime}\right)^{\prime}$ is a time-heterogenous affine Markov process. The closed form exponential affine expression of the conditional Laplace transform of process $\left(Y_{t}^{\prime}, F_{t}^{\prime}\right)^{\prime}$ simplifies the computation of the predictive distributions at any prediction horizon [see e.g. Darolles, Gourieroux, Jasiak (2006)] and the filtering of the latent factor [see Bates (2006)]. The closed form expression of the conditional Laplace transform also provides informative moment restrictions, which are the basis of estimation with the Generalized Method of Moments (see Section 3.4).

\subsection{A constrained stationary autoregressive Poisson model with gamma dy- namic frailty}

The dynamic Poisson model in equations (2.8) and (2.9) suffers from the curse of dimensionality, as it involves $K^{2} H^{2}$ contagion parameters to estimate. To solve this problem, we introduce constrained parametric specifications for the liquidation intensity. The general specification of Section 2.2 can be constrained by considering the following assumption:

Assumption A.3: The liquidation intensity is $\lambda_{k, h, t}(\theta)=\tilde{a}_{k}+\tilde{b}_{k} F_{t}+\tilde{c}_{k}^{\prime} Y_{t-1}$, where $Y_{t}=\left(Y_{1, t}, \ldots, Y_{K, t}\right)^{\prime}$ and $Y_{k, t}=\sum_{h=1}^{H} Y_{k, h, t}$.

Under Assumption A.3, the parameters vector $\theta$ consists of the intercept $\tilde{a}_{k}$, the frailty sensitivity $\tilde{b}_{k}$ and the vector of contagion coefficients $\tilde{c}_{k}$, for all management styles $k$. The effect of the lagged liquidation counts is passing through the liquidation counts aggregated over age classes. Moreover, the liquidation intensity at a given date $t$ is assumed independent of the fund age $h$, that is, it is equal 
across all funds of the same type. Nevertheless, the liquidation intensity of a given HF is time-varying as a result of the shared dynamic frailty and the liquidation counts history.

In some applications such as stress testing (see Section 3.6), it is appropriate to consider a given portfolio structure with respect to fund type and age, which is held fixed through time in the analysis.

Assumption A.4: The size adjustments are constant and equal to $1: \gamma_{k, h, t}=1$, for any $k, h, t$.

Thus, the sizes of the categories are fixed through time, and are assumed homogenous across fund type and age for expository purpose. Under Assumptions A.3-A.4 we can aggregate the liquidation counts over the $H$ age classes and consider the aggregate model:

$$
Y_{k, t} \sim \mathcal{P}\left(a_{k}+b_{k} F_{t}+c_{k}^{\prime} Y_{t-1}\right), \quad k=1, \ldots, K
$$

where $a_{k}=H \tilde{a}_{k}, b_{k}=H \tilde{b}_{k}$ and $c_{k}=H \tilde{c}_{k}$.

Assumption A.5: The frailty process $\left(F_{t}\right)$ is scalar $(m=1)$ and follows an Autoregressive Gamma $(A R G)$ process.

The ARG process is the time discretized Cox, Ingersoll, Ross process [Cox, Ingersoll, Ross (1985)]. The transition of this Markov process corresponds to a noncentral gamma distribution $\gamma\left(\delta, \eta F_{t-1}, \nu\right)$, where $\nu>0$ is a scale parameter, $\delta>0$ is the degree of freedom of the gamma transition distribution and parameter $\eta \geq 0$ is such that $\rho=\eta \nu$ is the first-order autocorrelation. The conditional Laplace transform is exponentially affine and given by equation (2.12) with:

$$
\alpha(u)=\frac{\eta \nu u}{1+\nu u}, \quad \beta(u)=\delta \log (1+\nu u), \quad u \geq 0
$$

(see Appendix 1 for basic results on the ARG process). Since the factor is unobservable, it is always possible to assume $E\left(F_{t}\right)=1$ for identification purpose. Then, the frailty dynamics can be conveniently parameterized by $\varphi=(\delta, \rho)^{\prime}$. 
The first and second-order unconditional moments of the liquidation counts process $Y_{t}$ are given in Proposition 1 below, which is proved in Appendix 2. Let vectors $a=\left(a_{1}, \ldots, a_{K}\right)^{\prime}$ and $b=\left(b_{1}, \ldots, b_{K}\right)^{\prime}$ gather the intercepts and frailty sensitivities for the $K$ management styles, and let $C=\left[c_{1}, \ldots, c_{K}\right]^{\prime}$ be the $(K, K)$ matrix whose rows correspond to the contagion coefficients for the $K$ management styles.

Proposition 1: In the Poisson model with Assumptions A.3-A.5, the joint process $\left(Y_{t}^{\prime}, F_{t}\right)^{\prime}$ is stationary if, and only if, $\rho<1$ and the eigenvalues of matrix $C$ are strictly smaller than 1 in modulus. Then, we have:

$$
E\left(Y_{t}\right)=(I d-C)^{-1}(a+b), \quad \operatorname{Cov}\left(Y_{t}, Y_{t-1}\right)=\sigma^{2} \rho b b^{\prime}\left(I d-\rho C^{\prime}\right)^{-1}+C V\left(Y_{t}\right)
$$

and the variance-covariance matrix $V\left(Y_{t}\right)$ is solution of the linear system:

$$
\begin{aligned}
V\left(Y_{t}\right)= & \operatorname{diag}\left[E\left(Y_{t}\right)\right]+C V\left(Y_{t}\right) C^{\prime}+\sigma^{2} b b^{\prime} \\
& +\sigma^{2} \rho C(I d-\rho C)^{-1} b b^{\prime}+\sigma^{2} \rho b b^{\prime}\left(I d-\rho C^{\prime}\right)^{-1} C^{\prime},
\end{aligned}
$$

where $\sigma^{2}=V\left(F_{t}\right)=1 / \delta$.

As expected, the stationarity condition of the joint process $\left(Y_{t}^{\prime}, F_{t}\right)^{\prime}$ requires both the stability of the contagion phenomenon, i.e. the eigenvalues of the contagion matrix $C$ are smaller than 1 in modulus, and the stationarity of the frailty process, i.e. the first-order autocorrelation coefficient $\rho$ is smaller than 1 . By expanding matrix $(I d-C)^{-1}$ in a power series, the expectation of the liquidation counts can be written as $E\left[Y_{t}\right]=(a+b)+C(a+b)+C^{2}(a+b)+\cdots$. The first term in the RHS $a+b$ is the expectation of the liquidation counts if there is no contagion, the second term $C(a+b)$ is the effect of contagion over a one-month period, the third term $C^{2}(a+b)$ is the indirect effect of contagion over a two-month period, etc. When the elements of matrix $C$ are all positive, the endogenous contagion amplifies the effect of the exogenous shared frailty on expected liquidation counts.

Equation (2.18) in Proposition 1 provides a decomposition of the historical variance-covariance matrix of the liquidation counts. The first term $\operatorname{diag}\left[E\left(Y_{t}\right)\right]$ in the right hand side of equation (2.18) 
corresponds to the variance in a Poisson model with cross-sectional independence. The sum of the first and second terms provides the expression of the variance in a model including contagion, but without frailty. The third term $\sigma^{2} b b^{\prime}$ captures the direct effect of the exogeneous frailty. The remaining terms in equation (2.18) accommodate its indirect effects through contagion, namely, the amplification of the frailty effect due to the network. This variance decomposition is written in an implicit form since the linear system (2.18) has to be solved to get the expression of $V\left(Y_{t}\right)$ as a function of the model parameters.

\section{Empirical analysis}

The dynamic Poisson model is applied to individual HF data from the Lipper TASS ${ }^{5}$ database.

\subsection{The Data}

The TASS database consists of monthly returns, Asset Under Management (AUM) and other HF characteristics for individual funds from February 1977 to June 2009. The relevant information for our study concerns the HF status. The database categorizes HF into "Live" and "Graveyard" funds. The "Live" funds are presented as still active. There are several reasons ${ }^{6}$ for a fund to be included in the Graveyard database. For instance, these funds $i$ ) no longer report their performance to TASS, $i i$ ) are liquidated, iii) are merged or restructured, iv) are closed to new investors. A HF can be listed in the Graveyard database only after being listed in the Live database. The TASS dataset includes 6097 funds in the "Live" database and 6767 funds in "Graveyard". In our analysis, we consider only the HF, which

\footnotetext{
${ }^{5}$ Tremont Advisory Shareholders Services. Further information about this database is provided on the website http://www.lipperweb.com/products/LipperTASS.aspx.

${ }^{6}$ Graveyard status code: 1 =fund liquidated; 2 =fund no longer reporting to TASS; $3=$ TASS unable to contact the manager for updated information; 4=fund closed to new investment; 5=fund has merged into another entity; 7=dormant fund; 9=unknown.
} 
are considered as "Live", or "Liquidated" (status code 1) ${ }^{7}$. The latter are 2533 funds. Moreover, in order to account for the time needed to pass from "Live" to "Graveyard" in TASS, we have transfered to the "Graveyard" database the 273 funds of the "Live" database with missing data at least for April, May, June 2009. Among these funds, 23 are considered as liquidated according to this criterion.

We apply a series of filters to the data. First, we have selected only funds with Net Asset Value (NAV) written in USD. This currency filter avoids double counting, since the same fund can have shares written in USD and EUR for example. After applying the currency code filter, we have 3183 funds in the "Live" base, and 1881 liquidated funds. Second, we have selected only funds with monthly reporting frequency. Nevertheless, we have also included the funds with quarterly reporting frequency, when the intermediate monthly estimated returns were available. Third, to keep the interpretation in terms of individual funds, we eliminate the funds of funds. Finally, in order to apply the Poisson approximation within the management styles, we select the management styles with a sufficiently large size. These are Long/Short Equity (LSE), Event Driven (ED), Managed Futures (MF), Equity Market Neutral (EMN), Fixed Income Arbitrage (FI), Global Macro (GM), Emerging Markets (EM), Multi Strategy (MS), and Convertible Arbitrage (CONV). After applying all these filters, we get 2279 funds in the "Live" database and 1520 liquidated funds. The distribution by style of alive and liquidated funds in the database is reported in Table 1.

[Insert Table 1: The database]

The largest management style in the database of alive and liquidated funds is Long/Short Equity Hedge (about 40\%), followed by Managed Futures, Multi-Strategy and Event Driven (each about 10\%).

The age of an individual HF is measured since the inception date reported in TASS. Thus, we do not take into account the incubation period preliminary to inception, and the possible associated left truncation bias.

\footnotetext{
${ }^{7}$ Chan, Getmansky, Haas, Lo (2007) have regarded as liquitaded all Graveyard funds in status code 1, 2 or 3.
} 


\subsection{Summary statistics}

In Figures 1 and 2 we provide the subpopulation sizes and the liquidation rates over time for different management styles, without distinguishing the age of the HF.

[Insert Figure 1: Subpopulation sizes of HF]

[Insert Figure 2: Liquidation rates of HF]

We observe in Figure 1 the HF market growth between 2000 and 2007, and the sharp decrease due to the 2008 financial crisis. However, the effect of the crisis is less pronounced for HF following a Global Macro strategy. Figure 2 shows liquidation clustering both with respect to time and among categories. One liquidation clustering due to the Long Term Capital Management (LTCM) debacle is observed in Summer 1998 and is especially visible for the Emerging Markets and Global Macro categories. Another liquidation clustering is observed in the 2008 crisis, but did not include the Global Macro strategy.

Let us now focus on the age effect. We provide in Figure 3 the smoothed nonparametric estimates of the liquidation intensity by management style. The estimates are obtained from the Kaplan-Meier estimators of the survival functions.

[Insert Figure 3: Smoothed estimates of liquidation intensity]

These estimates feature similar patterns, with a maximum at age of about 4 years. Table 2 provides the estimated liquidation intensities at the maximum, and at ages 0 and 100 months. We observe that the intensity functions of different management styles are not proportional. This suggests that the proportional hazard model should not be used for these data.

[Insert Table 2: Maximum and boundary values of the liquidation rates] 
There can also exist cross-effects of time and age in liquidation intensity, which are difficult to observe when the fund lifetimes are separately analysed w.r.t. either time (see Figure 2), or age (see Figure 3). These cross-effects can be detected by means of the Lexis diagram. Each liquidated fund is reported on the diagram by a dot with the date of death on the $x$-axis and its age at death on the $y$-axis. All the funds of the same cohort are represented by the $45^{0}$ line passing through this dot. In particular, the intersection of this line with the $x$-axis provides the birth date of the funds in this cohort (see Figure 4).

[Insert Figure 4: Lexis diagram]

The Lexis diagrams for four management styles are provided in Figures 5-8. In these figures, each star represents a liquidation event in the time-age plan, and we look for concentration of stars in a band either parallel to the $x$-axis (age effect), or parallel to the $y$-axis (time effect), or parallel to the $45^{0}$ line (cohort effect). For example, the Emerging Markets strategy represented in Figure 5 features a concentration of liquidation events around the age of 20 months, regularly spaced liquidation events for the cohort born in 1993, and another concentration during the crisis of 2008.

[Insert Figure 5: Lexis diagram for Emerging Markets]

The Lexis diagram for the Global Macro strategy (Figure 6) reveals two high time concentrations around 1998 (the LTCM crash) and 2008-2009 (the recent financial crisis), whereas the time concentrations are around January 2003 and the 2008 crisis for the Multi Strategy funds (Figure 7).

[Insert Figure 6: Lexis diagram for Global Macro]

[Insert Figure 7: Lexis diagram for Multi Strategy]

The strategy Managed Futures in Figure 8 shows essentially an age effect. 
We now move to the estimation of the dynamic Poisson model. To avoid the curse of dimensionality, we assume that the liquidation intensity is independent of age and that the lagged liquidation counts can be aggregated over age as in Section 2.3. To accommodate the HF market growth seen in Figure 1, we adjust for category sizes. The size adjustments are defined as follows: we rely on Assumption A.3 (Section 2.3) in which the aggregation scheme for the lagged counts is $Y_{t-1}^{*}=\left(Y_{1, t-1}^{*}, \ldots, Y_{K, t-1}^{*}\right)^{\prime}$, with $Y_{k, t-1}^{*}=Y_{k, t-1} / \gamma_{k, t-1}$ and $\gamma_{k, t-1}=\sum_{h=1}^{H} \gamma_{k, h, t-1}$. We select $\gamma_{k, h, t}$ as the ratio between the population size at month $t$, and the population size in February 2001, for type $k$ and age $h$. We consider first a model with pure contagion. Then we introduce also an unobserved frailty with autoregressive gamma dynamics (Assumption A.5 in Section 2.3).

\subsection{Model with contagion only}

Let us first focus on a model with pure contagion. As in Section 2.3, the data can be aggregated over the age to get:

$$
Y_{k, t} \sim \mathcal{P}\left[\gamma_{k, t}\left(a_{k}+c_{k}^{\prime} Y_{t-1}^{*}\right)\right], \quad k=1, \ldots, K
$$

This is a multivariate Poisson regression model, with $K=9$ lagged counts as explanatory variables [Cameron, Trivedi (1998)]. The lagged counts capture the liquidation clustering effects and their diffusion between management styles. The model involves 9 intercept parameters $a_{k}, k=1, \ldots, 9$, and a matrix of 81 contagion parameters $c_{k, k^{\prime}}$, with $k, k^{\prime}=1, \ldots, 9$. As usual the parameters of the Poisson regression model are estimated by the Maximum Likelihood (ML). As this model is a special case of Generalized Linear Model (GLM), the likelihood equations are easily solved by applying iteratively the weighted least squares in a Seemingly Unrelated Regression (SUR) model [McCullagh, Nelder (1989)]. The estimated values of the intercepts are given in Table 3 with standard errors in parentheses. The estimated contagion matrix is provided in Table 4, where we display only the statistically 
significant contagion coefficients at the $5 \%$ level.

[Insert Table 3: Estimated intercepts $a_{k}$ in the pure contagion model]

[Insert Table 4: Estimated contagion parameters $c_{k, k^{\prime}}$ in the pure contagion model]

The contagion matrix is represented as a network in Figure 9, where an arrow from style $k^{\prime}$ to style $k$ corresponds to a statistically significant estimate of parameter $c_{k, k^{\prime}}$.

[Insert Figure 9: The contagion scheme for the pure contagion model]

All strategies are interconnected either directly, or indirectly through multistep contagion channels. Such a contagion scheme corresponds to a complete structure in Allen, Gale (2000) terminology. The structure of the contagion matrix provides interesting information on the contagion interactions and the possible model misspecification. We observe the special roles of the Fixed Income Arbitrage and Long/Short Equity Hedge strategies, which both influence directly most of the other strategies. However, some estimated contagion parameters likely indicate a misspecification of the model without frailty and lead possibly to misleading interpretations. For instance, we get a large value 0.67 of the contagion parameter from Fixed Income Arbitrage to Long/Short Equity Hedge. Such a causal effect is unlikely since the Fixed Income Arbitrage strategies are investing in bonds and, when the associated managers deleverage their portfolios, the impact on Long/Short Equity strategies invested in stocks is expected to be small.

In Tables 3 and 4, the estimates of the intercepts $a_{k}$ and the rows $c_{k}^{\prime}$ of the contagion matrix differ significantly across management styles $k$. When a model with fund age and management style as the sole explanatory variables is fitted to the data, as in Figure 3 and Table 2, the variable age partly captures the effect of the time-varying lagged liquidation counts, that are the explanatory variables in model (3.1), with different impacts across the management styles. Thus, the results in Tables 3 and 4 
are compatible with the findings in Figure 3 and Table 2, and support the evidence that a proportional hazard specification without time-varying explanatory variables is not appropriate for our dataset.

\subsection{Model with frailty and contagion}

In the model with pure contagion, the funding and market liquidity risks cannot be separated and are both captured by the lagged liquidation counts. To disentangle the effects of the two types of liquidity risk, let us now extend the model to include an exogenous shared frailty with ARG dynamics representing funding liquidity risk (L-effect). We get:

$$
Y_{k, t} \sim \mathcal{P}\left[\gamma_{k, t}\left(a_{k}+b_{k} F_{t}+c_{k}^{\prime} Y_{t-1}^{*}\right)\right], \quad k=1, \ldots, K
$$

The single factor assumption is convenient for tractability. It is also in line with empirical findings in the literature. For instance, Carlson, Steinman (2008) regress the aggregate liquidation count for the entire HF market on several time-dependent observable variables related to market conditions and on the lagged aggregate liquidation count, and find only one statistically significant variable. ${ }^{8}$ The model in equation (3.2) involves 99 parameters $a_{k}, b_{k}$ and $c_{k, k^{\prime}}$ in the liquidation intensities, plus 2 parameters for the frailty dynamics, namely the degrees of freedom $\delta$ and the autocorrelation $\rho$. The large number of parameters is due to introducing cross-effects between management style and frailty, and between management style and lagged liquidation counts.

The likelihood function of this multivariate autoregressive Poisson regression model with shared dynamic frailty involves a multi-dimensional integral and a large number of parameters. This makes the numerical implementation of the likelihood maximization cumbersome. We propose below an

\footnotetext{
${ }^{8}$ These market conditions are essentially the market index return and the market volatility, the significant observed variable being the S\&P 500 return. However, the model in Carlson, Steinman (2008) includes no variable measuring liquidity features, such as measures of counterparty risk. In the discussion of the estimated contagion effects we see that the effect of the S\&P 500 return is likely a reduced form effect, that is a consequence of the subprime crisis and the L-effect.
} 
informative Generalized Method of Moments (GMM) approach for our estimation problem. The moment restrictions can involve either the stationary distribution of the frailty (static moment restrictions), or the transition distribution of the frailty (dynamic moment restrictions).

\section{i) Static moment restrictions}

The moment restrictions are based on the special form of the conditional Laplace transform in equation (2.11). For type $k$ and by aggregating over the age $h$, we have:

$$
E\left[\exp \left(-u_{k} Y_{k, t}\right) \mid \underline{F_{t}}, \underline{Y_{t-1}}\right]=\exp \left\{-\gamma_{k, t}\left(a_{k}+b_{k} F_{t}+c_{k}^{\prime} Y_{t-1}^{*}\right)\left(1-e^{-u_{k}}\right)\right\}
$$

for any argument $u_{k} \in[0, \infty)$. These conditional moments are appropriate for analyzing risk parameters. Indeed, the left hand side of the above equation is simply the expected utility function for an investor with a portfolio totally invested in the liquidation events of style $k$, and an absolute risk aversion equal to $u_{k}$. By considering the associated set of moment restrictions, we consider all types of investment, for all values of risk aversion. Therefore, the associated moment method will calibrate the unknown parameters on the whole set of expected utilities.

The equations in (3.3) can be rewritten as:

$$
E\left[\exp \left\{-u_{k} Y_{k, t}+\gamma_{k, t}\left(a_{k}+c_{k}^{\prime} Y_{t-1}^{*}\right)\left(1-e^{-u_{k}}\right)\right\} \mid \underline{F_{t}}, \underline{Y_{t-1}}\right]=\exp \left\{-\gamma_{k, t} b_{k}\left(1-e^{-u_{k}}\right) F_{t}\right\}
$$

for $u_{k} \in[0, \infty)$. Thus, we obtain nonlinear transforms of the observable liquidation count variables, whose conditional expectation depends on the frailty only. As equation (3.4) holds for all real positive arguments $u_{k}$, we can consider a time-dependent argument $u_{k}$. The time dependence is selected such that the RHS of equation (3.4), and thus the LHS as well, is stationary. More precisely, let $u_{k, t}$ be such that $1-e^{-u_{k, t}}=v / \gamma_{k, t}$, for given $v \in \mathcal{V}_{k}$, i.e. $u_{k, t}=-\log \left(1-v / \gamma_{k, t}\right)$. In order to obtain a well-defined $u_{k, t}$ in $[0, \infty)$, the real interval $\mathcal{V}_{k}$ has to be a subset of $\left[0, \inf _{t} \gamma_{k, t}\right)$. Then, equation (3.4) becomes:

$$
E\left[\exp \left\{\log \left(1-v / \gamma_{k, t}\right) Y_{k, t}+v\left(a_{k}+c_{k}^{\prime} Y_{t-1}^{*}\right)\right\} \mid \underline{F_{t}}, \underline{Y_{t-1}}\right]=\exp \left(-v b_{k} F_{t}\right), \quad \forall v \in \mathcal{V}_{k}
$$


These moment restrictions are conditional on factor path $\underline{F_{t}}$ and cannot be used directly for estimation since the factor is unobservable. Therefore, we integrate out the latent factor by taking expectation on both sides of the equation w.r.t. the gamma stationary distribution $\gamma(\delta, 0,1 / \delta)$ of factor process $F_{t}$ (see Appendix 1). We get a continuum of unconditional moment restrictions:

$$
E\left[\exp \left\{\log \left(1-v / \gamma_{k, t}\right) Y_{k, t}+v\left(a_{k}+c_{k}^{\prime} Y_{t-1}^{*}\right)\right\}\right]=\frac{1}{\left(1+v b_{k} / \delta\right)^{\delta}}, \quad \forall v \in \mathcal{V}_{k}
$$

These static moment restrictions involve the intensity parameters $a_{k}, b_{k}, c_{k}$ for any type $k$, as well as parameter $\delta$ characterizing the stationary distribution of the frailty, but do not allow to identify the frailty persistence parameter $\rho$.

\section{ii) Dynamic moment restrictions}

In order to derive moment restrictions that allow for estimation of parameter $\rho$, let us consider equation (3.5) and multiply both sides by $\exp \left(-\tilde{u}_{l, t-1} Y_{l, t-1}\right)$, where $\tilde{u}_{l, t-1}=-\log \left(1-\tilde{v} / \gamma_{l, t-1}\right)$, for some type $l$ and any $\tilde{v} \in \mathcal{V}_{l}$. We have:

$$
E\left[\exp \left\{-u_{k, t} Y_{k, t}-\tilde{u}_{l, t-1} Y_{l, t-1}+v\left(a_{k}+c_{k}^{\prime} Y_{t-1}^{*}\right)\right\} \mid \underline{F_{t}}, \underline{Y_{t-1}}\right]=\exp \left(-v b_{k} F_{t}-\tilde{u}_{l, t-1} Y_{l, t-1}\right)
$$

for all $v \in \mathcal{V}_{k}, \tilde{v} \in \mathcal{V}_{l}$, where $u_{k, t}=\log \left(1-v / \gamma_{k, t}\right)$. By taking the conditional expectation given $\underline{F_{t}}$ and the liquidation counts history $\underline{Y_{t-2}}$ up to month $t-2$ on both sides of the equation, we get:

$$
\begin{aligned}
& E\left[\exp \left\{-u_{k, t} Y_{k, t}-\tilde{u}_{l, t-1} Y_{l, t-1}+v\left(a_{k}+c_{k}^{\prime} Y_{t-1}^{*}\right)\right\} \mid \underline{F_{t}}, \underline{Y_{t-2}}\right] \\
= & \exp \left(-v b_{k} F_{t}\right) E\left[\exp \left(-\tilde{u}_{l, t-1} Y_{l, t-1}\right) \mid \underline{F_{t}}, \underline{Y_{t-2}}\right]=\exp \left\{-v b_{k} F_{t}-\left(a_{l}+b_{l} F_{t-1}+c_{l}^{\prime} Y_{t-2}^{*}\right) \tilde{v}\right\} .
\end{aligned}
$$

By rearranging terms, and computing the unconditional expectation of both sides, we get:

$E\left[\exp \left\{-u_{k, t} Y_{k, t}-\tilde{u}_{l, t-1} Y_{l, t-1}+v\left(a_{k}+c_{k}^{\prime} Y_{t-1}^{*}\right)+\tilde{v}\left(a_{l}+c_{l}^{\prime} Y_{t-2}^{*}\right)\right\}\right]=E\left[\exp \left(-v b_{k} F_{t}-\tilde{v} b_{l} F_{t-1}\right)\right]$

for all $v \in \mathcal{V}_{k}, \tilde{v} \in \mathcal{V}_{l}$. The expectation in the right-hand side involves the joint distribution of the frailty values $F_{t}$ and $F_{t-1}$ on two consecutive months, and hence depends on the frailty autocorrelation 
parameter $\rho$. In fact, by the exponential affine property of the ARG process we have:

$$
\begin{aligned}
& E\left[\exp \left(-v b_{k} F_{t}-\tilde{v} b_{l} F_{t-1}\right)\right] \\
= & E\left[E\left[\exp \left(-v b_{k} F_{t}\right) \mid F_{t-1}\right] \exp \left(-\tilde{v} b_{l} F_{t-1}\right)\right]=\exp \left(-\beta\left(v b_{k}\right)\right) E\left[\exp \left(-\left[\alpha\left(v b_{k}\right)+\tilde{v} b_{l}\right] F_{t-1}\right)\right] \\
= & \frac{1}{\left[1+(1-\rho) v b_{k} / \delta\right]^{\delta}} \frac{1}{\left[1+\left[\alpha\left(b v_{k}\right)+\tilde{v} b_{l}\right] / \delta\right]^{\delta}}=\frac{1}{\left[1+\left(v b_{k}+\tilde{v} b_{l}\right) / \delta+(1-\rho) v \tilde{v} b_{k} b_{l} / \delta^{2}\right]^{\delta}},
\end{aligned}
$$

where we use $\beta(u)=\delta \log (1+(1-\rho) u / \delta)$ and $\alpha(u)=\frac{\rho u}{1+(1-\rho) u / \delta}$ from equations (2.16) and (a.3) in Appendix 1. Thus, we get the continuum of unconditional dynamic moment restrictions:

$$
\begin{aligned}
& E\left[\exp \left\{\log \left(1-v / \gamma_{k, t}\right) Y_{k, t}+\log \left(1-\tilde{v} / \gamma_{l, t-1}\right) Y_{l, t-1}+v\left(a_{k}+c_{k}^{\prime} Y_{t-1}^{*}\right)+\tilde{v}\left(a_{l}+c_{l}^{\prime} Y_{t-2}^{*}\right)\right\}\right] \\
= & \frac{1}{\left[1+\left(v b_{k}+\tilde{v} b_{l}\right) / \delta+(1-\rho) v \tilde{v} b_{k} b_{l} / \delta^{2}\right]^{\delta}}, \quad \forall v \in \mathcal{V}_{k}, \tilde{v} \in \mathcal{V}_{l} .
\end{aligned}
$$

These dynamic moment restrictions, written for all pairs of management styles $(k, l)$, involve all model parameters. For $\tilde{v}=0$, these moment restrictions reduce to the static moment restrictions (3.6).

\section{iii) GMM estimators}

The above moment restrictions can be used is different ways to define GMM estimators. We consider below three possible GMM approaches.

a) We can use the static moment restrictions (3.6) written for all types $k$ to define a first-step GMM estimator of parameters $a_{k}, b_{k}, c_{k}$, for all management styles $k$, and parameter $\delta$. Then, a secondstep GMM estimator of parameter $\rho$ can be defined by using some subset of the dynamic moment restrictions (3.7) and replacing parameters $a_{k}, b_{k}, c_{k}$ and $\delta$ with their first-step GMM estimates.

b) Alternatively, the dynamic moment restrictions (3.7) can be used to estimate jointly all model parameters $\left(\theta^{\prime}, \varphi^{\prime}\right)^{\prime}$, where $\theta=\left(a_{k}, b_{k}, c_{k}^{\prime}, k=1, \ldots, K,\right)^{\prime}$ and $\varphi=(\delta, \rho)^{\prime}$, in one step. More precisely, consider the dynamic moment restrictions (3.7) written for all management styles $k$, with $l=k$, and for a given grid of values $v, \tilde{v} .9$ They define a set of unconditional moment restric-

\footnotetext{
${ }^{9}$ It might also be possible to apply an efficient GMM taking into account the continuum of moment restrictions (3.7) [see e.g. Carrasco et al (2007)].
} 
tions $E\left[h_{k, t}\left(a_{k}, b_{k}, c_{k}, \varphi\right)\right]=0$, for $k=1, \ldots, K$, say. Then, the GMM estimator $\left(\hat{\theta}^{\prime}, \hat{\varphi}^{\prime}\right)^{\prime}$ minimizes the criterion:

$$
Q_{T}(\theta, \varphi)=\sum_{k=1}^{K}\left\|\hat{h}_{k, T}\left(a_{k}, b_{k}, c_{k}, \varphi\right)\right\|^{2}
$$

where $\hat{h}_{k, T}\left(a_{k}, b_{k}, c_{k}, \varphi\right)=\frac{1}{T} \sum_{t=1}^{T} h_{k, t}\left(a_{k}, b_{k}, c_{k}, \varphi\right)$ is the sample average of the orthogonality function. By including only the moment restrictions with $k=l$, and using a weighting matrix that is diagonal across management styles, we simplify considerably the optimization problem. ${ }^{10}$ In fact, for given value of the bivariate frailty parameter $\varphi=(\delta, \rho)^{\prime}$, the GMM criterion (3.8) is additive w.r.t. the parameters of the styles. Therefore, the minimization can be performed by concentrating w.r.t. parameter $\theta$. Namely, for given $\varphi$, the criteria for the different styles $k$ are separately minimized w.r.t. parameters $a_{k}, b_{k}, c_{k}$ by a Newton-Raphson algorithm. Then, the concentrated criterion is minimized by a bi-dimensional grid search over parameters $(\delta, \rho)$.

c) We can also consider the GMM estimators that exploit the full set of dynamic moment restrictions (3.7) written for all pairs of management styles $(k, l)$. Such GMM estimators are expected to be more efficient than the GMM estimator minimizing criterion (3.8). However, such GMM estimators are computationally less convenient, because they involve an optimization over a large-dimensional parameter space that can not be dealt with by concentration easily. In this paper we apply method $b$ ), which provides a trade-off between tractability and efficiency.

\section{iv) Estimation results}

In Table 5 we display the estimated intercept parameters $a_{k}$ and factor sensitivities $b_{k}$ along with their standard errors. The estimated contagion matrix is provided in Table 6, where we display only the statistically significant contagion coefficients at the $10 \%$ level. The estimated parameters of the frailty dynamics are $\hat{\delta}=0.59$ (with standard error 0.34 ) and $\hat{\rho}=0.74$ (with standard error 0.20 ). To simplify the numerical computation of the GMM estimator, we set equal to zero the parameters in the

\footnotetext{
${ }^{10}$ For expository purpose the weighting matrix is set equal to the identity matrix for each type.
} 
contagion matrix whose estimates in the pure contagion model are not statistically significant. The standard errors of the GMM estimates are computed using the asymptotic distribution.

[Insert Table 5: Estimated intercepts $a_{k}$ and factor sensitivities $b_{k}$ in the model with contagion and frailty]

[Insert Table 6: Estimated contagion parameters $c_{k, k^{\prime}}$ in the model with contagion and frailty]

The estimated factor sensitivities are all positive and statistically significant. As discussed in the introduction, the unobservable frailty is likely representing a measure of funding liquidity risk. This is confirmed by a careful analysis of the estimated sensitivities. For a given management style, the liquidity features are twofold: the portfolio can be invested in more or less liquid assets, and the strategy can require a longer or shorter horizon to be applied. In this respect, Long/Short Equity Hedge portfolios are invested in very liquid assets, and then can offer to investors weekly, or even daily liquidity conditions, and thus have a large sensitivity coefficient 4.55. At the opposite, the Fixed Income Arbitrage strategies are based on arbitrages with high leverage requiring a long horizon to perform, and have less interesting, generally quarterly, liquidity conditions; the factor sensitivity coefficient 0.31 is the smallest one. Similar remarks can be done for other management styles. For instance, the Event Driven strategies are essentially looking for positive outcomes in merger and acquisitions, which can only be expected in a medium horizon.

The sums $a_{k}+b_{k}=a_{k}+b_{k} E\left[F_{t}\right]$ in Table 5 are much larger than the coefficients $a_{k}$ estimated in the pure contagion model (see Table 3). Thus, a large fraction of the common liquidation features is captured by the introduction of the frailty. This effect is balanced by a diminution in the estimated contagion parameters. Only 11, resp. 13, estimated contagion parameters in Table 6 are statistically significant at $5 \%$, resp. $10 \%$, level. These numbers have to be compared with the 27 statistically significant contagion parameters at $5 \%$ in Table 4 obtained when the frailty component is omitted. The 
contagion scheme for the model including both contagion and frailty is displayed in Figure 10, where any estimated contagion coefficient in Table 6 , that is statistically significant at $5 \%$ level, is represented by an arrow.

[Insert Figure 10: The contagion scheme for the model with contagion and frailty]

By comparing Figure 10 with Figure 9, as expected the introduction of the systematic risk factor $F_{t}$ largely diminishes the perception of contagion phenomena. In particular, we observe that the key roles of Fixed Income Arbitrage and Long-Short Equity Hedge in Table 4 and Figure 9 were mostly due to the effect of the common factor, and the fact that these management strategies are rather sensitive to the factor, especially the second management style. Moreover, in Figure 10 we observe that contagion occurs along some specific directions, such as Multi Strategy $\rightarrow$ Equity Market Neutral $\rightarrow$ Event Driven $\rightarrow$ Fixed Income Arbitrage $\rightarrow$ Emerging Markets, without any evidence of contagion in the reverse direction.

Let us now discuss carefully the scheme in Figure 10. We observe four types of funds:

i) Funds mainly invested in fixed income products and using high leverage, that are Fixed Income Arbitrage, Managed Futures, Emerging Markets and Global Macro.

ii) Funds mainly invested in equities, such as Equity Market Neutral, Long/Short Equity Hedge and Event Driven.

iii) Funds in the Convertible Arbitrage management style, in which the convertible products have features of the corporate bonds and associated stocks.

iv) Funds in the Multi-Strategy management style, with portfolios including subprimes and equities. Then, the causal scheme is likely due to the subprime crisis and the associated lack of market liquidity on the different classes of assets. In 2007, there has been an increase of the expected default rates for mortgages, and then an increase of margin calls for credit derivatives. The Multi-Strategy funds, largely invested in subprimes but also on liquid market neutral strategies, had to get cash in order to 
satisfy these calls. It was then natural for them to liquidate the most liquid part of their portfolios, i.e. the equity strategies. This massive deleveraging had a direct effect on stock prices. At the beginning this effect cannot be observed on the stock indices, but mainly on the relative performances of the individual stocks: the high-ranked stocks becoming low ranked and vice-versa, since the strategies followed by Equity Market Neutral and Long/Short Equity Hedge funds, for instance, are orthogonal to the market. This dislocation effect on stock prices has impacted all the equity strategies, including the Event Driven funds. The associated M\&A strategies have transformed the short-term shocks into long-term shocks. This explains the key (systemic) role of the Event Driven management style, which creates the link between the shocks on stock markets and the shocks on fixed income markets.

The relative effect of contagion and frailty on liquidation risk can be measured by using the variance decomposition derived in Proposition 1. Let us consider a portfolio of Liquidation Swaps (LS) written on the individual hedge funds, which is diversified with respect to the types in order to ensure $\gamma_{k, t}=1$, for all $k, t$. The liquidation swap for management style $k$ pays 1 USD for each fund of style $k$ that is liquidated in month $t$. The payoff of the LS portfolio at month $t$ is $e^{\prime} Y_{t}$, where $e=(1,1, \ldots, 1)^{\prime}$ is a $(K, 1)$ vector of ones. To ensure the time-invariant diversification, the portfolio of LS has to be appropriately rebalanced when a liquidation occurs. By using the decomposition of variance in Proposition 1, we can evaluate the percentage of portfolio variance $e^{\prime} V\left(Y_{t}\right) e$, due to the underlying Poisson shocks, contagion and frailty, respectively. We have the following decomposition:

\begin{tabular}{c|c|c|c|c}
\hline \hline & Underlying Poisson & Contagion & Frailty (direct) & Frailty (indirect) \\
\hline Percentage of variance & $6.54 \%$ & $5.10 \%$ & $64.30 \%$ & $24.06 \%$ \\
\hline \hline
\end{tabular}

The largest contribution to the portfolio variance comes from the frailty process, either through a direct effect $(64.30 \%)$, or through an indirect effect via the contagion network (24.06\%). The remaining part of portfolio variance is explained by the underlying Poisson shocks (6.54\%) and the direct contagion effects $(5.10 \%)$. Eventhough the direct effect of contagion is modest, the network plays an important 
role in amplifying the effect of the exogenous frailty.

\subsection{Diagnostic checks for shared frailty}

In this section we discuss diagnostic tools to test for the presence of the shared frailty effect. Our aim is to provide empirical evidence that the Poisson model with contagion only is not able to reproduce some relevant features in the joint dynamics of the liquidation counts.

As it is common in tests of omitted heterogeneity, it is difficult to establish an optimal testing procedure and, when it exists, it is complicated to implement it in practice. The difficulties are the following:

i) The null hypothesis of no frailty effect can be written as:

$$
\mathcal{H}_{0}:\left\{\sigma^{2}=0\right\} \cup\left\{b_{k}=0, \forall k\right\}
$$

where $\sigma^{2}=V\left(F_{t}\right)$ is the frailty variance. Thus, we have no heterogeneity effect, if either the heterogeneity is constant through time, or the sensitivity coefficients $b_{k}$ are all equal to $0 .{ }^{11}$ The null hypothesis is the union of two subspaces of the parameter set. It is nonstandard, especially as the dimensions, and thus the degrees of freedom, are not the same on $\left\{\sigma^{2}=0\right\}$, on $\left\{b_{k}=0, \forall k\right\}$, and on the intersection $\left\{\sigma^{2}=0\right\} \cap\left\{b_{k}=0, \forall k\right\}$.

ii) The null hypothesis $\left\{\sigma^{2}=0\right\}$ touches the boundary of the parameter set, since $\sigma^{2} \geq 0$.

iii) Finally, under the null hypothesis we have $Y_{k, t} \sim \mathcal{P}\left(\gamma_{k, t} \lambda_{k, t}^{0}\right)$ conditionally on $Y_{t-1}$, where $\lambda_{k, t}^{0}=a_{k}+b_{k}+c_{k}^{\prime} Y_{t-1}^{*}$. Thus, the identifiable parameters are $a_{k}+b_{k}$ and $c_{k}$, for $k=1, \ldots, K$. In

\footnotetext{
${ }^{11}$ Das et al. (2007) and Lando, Nielsen (2010) test for default independence across U.S. corporations conditional on a given set of observable common and firm-specific variables (doubly stochastic assumption). In Das et al. (2007) and Lando, Nielsen (2010), the null hypothesis is a model with common factors and without contagion, while in our paper the null hypothesis is a model with contagion and without common factors. Moreover, common factors are assume observable in Das et al. (2007) and Lando, Nielsen (2010), while they are unbservable in our paper.
} 
particular, the sensitivities $b_{k}, k=1, \ldots K$, the variance $\sigma^{2}$ and the frailty autocorrelation $\rho$ are not identifiable under the null hypothesis.

It is complicated to develop optimal tests when some parameters, here the $b_{k}, \sigma^{2}$ and $\rho$, are not identifiable under the null [see e.g. Andrews, Ploberger (1994)]. However, it is easy to construct informative diagnostic tools. For this purpose, we consider the score w.r.t. parameter $\sigma^{2}$, namely the partial derivative of the log-likelihood function w.r.t. $\sigma^{2}$, evaluated at the true parameter value satisfying the null hypothesis $\mathcal{H}_{0}$ of no frailty effect. ${ }^{12}$ This score is given in equation (a.10) in Appendix 3 . It involves the unknown parameters $a_{k}+b_{k}, b_{k}, c_{k}$ and $\rho$. In the standard use of such a score, these parameters would have to be replaced by their constrained ML estimators. In our framework, the constrained estimates of parameters $b_{k}$ and $\rho$ are not uniquely defined, due to the lack of identification of these parameters under the null. Nevertheless, the expression of the score in equation (a.10) suggests some basic statistics, which can be used to construct diagnostic tools. These are:

$$
\begin{aligned}
\xi_{k l, T} & =\frac{1}{T} \sum_{t=1}^{T} \gamma_{k, t} \gamma_{l, t}\left[\frac{\partial^{2} \log p\left(Y_{k, t} ; \gamma_{k, t} \lambda_{k, t}^{0}\right)}{\partial \lambda^{2}} \mathbf{1}\{k=l\}+\frac{\partial \log p\left(Y_{k, t} ; \gamma_{k, t} \lambda_{k, t}^{0}\right)}{\partial \lambda} \frac{\partial \log p\left(Y_{l, t} ; \gamma_{l, t} \lambda_{l, t}^{0}\right)}{\partial \lambda}\right] \\
& =\frac{1}{T} \sum_{t=1}^{T} \gamma_{k, t} \gamma_{l, t}\left[\left(\frac{Y_{k, t}}{\gamma_{k, t} \lambda_{k, t}^{0}}-1\right)\left(\frac{Y_{l, t}}{\gamma_{l, t} \lambda_{l, t}^{0}}-1\right)-\frac{Y_{k, t}}{\left(\gamma_{k, t} \lambda_{k, t}^{0}\right)^{2}} \mathbf{1}\{k=l\}\right],
\end{aligned}
$$

and:

$$
\begin{aligned}
r_{k l, T}(s) & =\frac{1}{T} \sum_{t=s+1}^{T} \gamma_{k, t} \gamma_{l, t-s} \frac{\partial \log p\left(Y_{k, t} ; \gamma_{k, t} \lambda_{k, t}^{0}\right)}{\partial \lambda} \frac{\partial \log p\left(Y_{l, t-s} ; \gamma_{l, t-s} \lambda_{l, t-s}^{0}\right)}{\partial \lambda} \\
& =\frac{1}{T} \sum_{t=s+1}^{T} \gamma_{k, t} \gamma_{l, t-s}\left(\frac{Y_{k, t}}{\gamma_{k, t} \lambda_{k, t}^{0}}-1\right)\left(\frac{Y_{l, t-s}}{\gamma_{l, t-s} \lambda_{l, t-s}^{0}}-1\right),
\end{aligned}
$$

for $k, l=1, \ldots, K$ and $s=1,2, \ldots$, where $p(\cdot ; \lambda)$ is the p.d.f. of the Poisson distribution with intensity parameter $\lambda$. The statistic $\xi_{k l, T}$ corresponds to the standard information-matrix test [White (1982)] for the pair $(k, l)$, which is a score test for omitted heterogeneity [Chesher (1984)]. For a Poisson regression model, statistic $\xi_{k l, T}$ with $k=l$ corresponds to a test for conditional overdispersion, that

\footnotetext{
${ }^{12}$ From the expression of the null hypothesis, we might also consider the score w.r.t. $b_{k}$ under the null. However, it is equal to the score w.r.t. $a_{k}$, due to the identification problem, and is not informative.
} 
is, a test for the null hypothesis that the mean and the variance of the conditional distribution of liquidation counts in management style $k$ are equal. Indeed, statistic $\xi_{k k, T}$ involves the difference between the weighted mean and variance of the liquidation counts for management style $k$. It is well known that the Poisson distribution features no overdispersion, that is, the mean is equal to the variance. In the statistical literature, overdispersion in count data is typically modeled my means of unobservable heterogeneities [see e.g. Cox (1983)]. In the dynamic model estimated in Section 3.4, overdispersion in the conditional distribution of liquidation counts is generated by the shared dynamic frailty. For $k \neq l$, statistic $\xi_{k l, T}$ corresponds to a weighted covariance between the residuals of the Poisson regressions $Y_{k, t} /\left(\gamma_{k, t} \lambda_{k, t}^{0}\right)-1$ and $Y_{l, t} /\left(\gamma_{l, t} \lambda_{l, t}^{0}\right)-1$ for styles $k$ and $l$, and can be used to test for the absence of conditional contemporaneous correlation across styles. The values of statistics $\xi_{k l, T}$ are symmetric with respect to the indices $k$ and $l$, that is, $\xi_{k l, T}=\xi_{l k, T}$. The statistics $r_{k l, T}(s)$ are used to detect possible serial auto- and cross-correlation between the residuals of the Poisson regression model. Indeed, if the model without shared dynamic frailty is correctly specified, the Poisson residuals feature no serial auto- and cross-correlation.

These basic statistics can be used as diagnostic tools. We construct the standardized statistics:

$$
\xi_{k l, T}^{*}=\xi_{k l, T} / \sqrt{\hat{\omega}_{k l, T}}, \quad k, l=1, \ldots, K
$$

where $\hat{\omega}_{k l, T}$ is an estimate of the asymptotic variance of $\xi_{k l, T}$. Statistic $\xi_{k l, T}^{*}$ is asymptotically standard Gaussian distributed under the null hypothesis $\mathcal{H}_{0}$ of no frailty effect. The values of $\xi_{k l, T}^{*}$ are displayed as a $(K, K)$ array in Table 7.

[Insert Table 7: Statistics $\xi_{k l, T}^{*}$ for diagnostic check of conditional overdispersion and conditional contemporaneous cross-correlation ]

When the shared frailty is not introduced in the model, five HF management styles feature conditional overdispersion in liquidation counts. Moreover, we find evidence of conditional contemporaneous 
cross-correlation in Poisson residuals, in particular between Long/Short Equity Hedge and all other management styles except Convertible Arbitrage.

For each pair $(k, l)$, we also compute the estimated (cross-)ACF of the Poisson residuals:

$$
r_{k l, T}^{*}(s)=r_{k l, T}(s) / \sqrt{r_{k k, T}(0) r_{l l, T}(0)}, \quad s=1,2, \ldots
$$

with their confidence bands to detect if there is some omitted dynamic in the pure contagion model. The (cross-)ACF for management styles Fixed Income Arbitrage, Global Macro and Long/Short Equity Hedge are displayed in Figure 11.

[Insert Figure 11: (Cross-)ACF $r_{k l, T}^{*}(s)$ for diagnostic check]

We find evidence of autocorrelation in the Poisson residuals of the Long/Short Equity Hedge style, as well as cross-correlation between the residuals of the three management styles, especially between residuals of Long/Short Equity Hedge and leads and lags of residuals of Fixed Income Arbitrage.

Overall, the results in Table 7 and Figure 11 show that the Poisson model with contagion only does not fully capture the serial and cross-sectional dependencies featured by the joint dynamics of the liquidation counts. These findings suggest that it is necessary to introduce a dynamic frailty in the autoregressive Poisson model to achive a satisfactory specification.

\subsection{Stress-tests}

The estimated model with dynamic frailty and contagion can be used for portfolio management of a fund of funds, for computation of reserves, etc. In this section we illustrate how to implement stresstests for liquidation risk. We consider a portfolio of HF with fixed category sizes such that $\gamma_{k, t}=1$, for all $k$ and $t$. The stress can be designed as follows:

i) We can stress the current factor value by setting $F_{t}=q_{\alpha}$ in the conditioning set, where $q_{\alpha}$ is the quantile of the estimated stationary distribution of the frailty $F_{t}$ at level $\alpha$. By choosing $\alpha=95 \%$, or 
$99 \%$, we consider an extreme scenario with a large transitory shock on the underlying funding liquidity risk factor at month $t$.

ii) We can change some parameters values, by either "increasing" the matrix of contagion, or by increasing the value of the frailty persistence parameter $\rho$. This stress scenario will increase the liquidation risks by amplifying the impact of the exogenous shock by contagion and by introducing some exogenous shocks clustering, respectively.

For any given stress scenario $F_{t}=q_{\alpha^{s}}$ and $\left(\theta^{s}, \varphi^{s}\right)$, where $\alpha^{s}$ and $\left(\theta^{s}, \varphi^{s}\right)$ denote the frailty quantile level and the model parameters values in the stress scenario, we compute the term structure of expected liquidation counts, volatility and overdispersion of these counts, for the different types:

$$
\tau \rightarrow\left(\underset{\left(\theta^{s}, \varphi^{s}\right)}{E}\left(Y_{k, t+\tau} \mid F_{t}=q_{\alpha^{s}}, Y_{t}\right), \underset{\left(\theta^{s}, \varphi^{s}\right)}{V}\left(Y_{k, t+\tau} \mid F_{t}=q_{\alpha^{s}}, Y_{t}\right)^{1 / 2}, \frac{\underset{\left(\theta^{s}, \varphi^{s}\right)}{V}\left(Y_{k, t+\tau} \mid F_{t}=q_{\alpha^{s}}, Y_{t}\right)}{\underset{\left(\theta^{s}, \varphi^{s}\right)}{E}\left(Y_{k, t+\tau} \mid F_{t}=q_{\alpha^{s}}, Y_{t}\right)}\right)
$$

where $\tau=1,2, \ldots$ is the horizon, and $\underset{\left(\theta^{s}, \varphi^{s}\right)}{E}$ and $\underset{\left(\theta^{s}, \varphi^{s}\right)}{V}$ denote conditional mean and variance computed for parameter values $\left(\theta^{s}, \varphi^{s}\right)$. These conditional moments are derived in closed form by using the exponential affine property of the joint process of frailty and liquidation counts (see Sections 2.2 and 2.3, and the supplementary materials). By conditioning on an extreme event, these statistics are in line with the measures for systemic risk as the CoVaR [Adrian, Brunnermeier (2011)], or the marginal expected shortfall [Acharya et al. (2010)]. The main difference is in the definition of the conditioning set including the unobservable factor and the observable liquidation counts, instead of including the return of a market portfolio. Sialm, Sun and Zheng (2012) use CoVaR to measure contagion in returns of funds of funds.

Our stress test analysis is dynamic, as it fully accounts for both liquidation counts and exogenous frailty dynamics. Therefore, it sharply differs from the stress test analysis in models with time-varying observable variables, in which a crystallized scenario for the future factor path is assumed. Such a stress test would neglect the liquidation risk dependence induced by the exogenous factors.

We consider three sets of stress scenarios: 
S.1: The current factor value $F_{t}$ is increased from the median, i.e. $\alpha^{s}=0.50$, to the $95 \%$ quantile, i.e. $\alpha^{s}=0.95$, of the historical distribution. Parameter values $\left(\theta^{s}, \varphi^{s}\right)$ correspond to the estimates of Section 3.4.

S.2: The contagion matrix is changed from $C^{s}=\hat{C}$ to $C^{s}=2 \hat{C}$, where $\hat{C}$ is the estimate of Section 3.4. The other parameter values are kept constant, equal to the estimates of Section 3.4. The current factor value $F_{t}$ is the median of the historical distribution.

S.3: The frailty autocorrelation is increased from $\rho^{s}=0.74$ (corresponding to the estimate in Section 3.4) to $\rho^{s}=0.90$. The other parameter values are kept constant, equal to the estimates of Section 3.4. The current factor value $F_{t}$ is the median of the historical distribution.

For all stress scenarios, the vector of liquidation counts $Y_{t}$ in the conditioning set is equal to the observations on the liquidation counts in the last month of the sample, i.e. June 2009. In Figures 12 , 13 and 14 we display for the nine management styles the impact of stress scenarios S.1, S.2 and S.3, respectively, on the term structures of the conditional expectations of liquidation counts. ${ }^{13}$

[Insert Figure 12: Term structure of expected liquidation counts when stressing the current factor value]

[Insert Figure 13: Term structure of expected liquidation counts when stressing the contagion matrix]

[Insert Figure 14: Term structure of expected liquidation counts when stressing the frailty persistence]

In each figure, the squares represent the term structures of expected liquidation counts before stress, that are the same in each scenario. As the horizon increases, the term structure converges to the unconditional expectation of the liquidation count, for each management style. The term structures are upward sloping since the current month, i.e. June 2009, corresponds to a period with few liquidation

\footnotetext{
${ }^{13}$ Figures with the term structures of conditional volatility and overdispersion of liquidation counts are provided in the supplementary material.
} 
events compared to the historical average in any style. The circles represent the term structures of expected liquidation counts after the shock. We observe that the three types of shocks have very different effects on the term structures. The shock to the factor value in stress scenario S.1 is a transitory funding liquidity shock, with different impacts in the short run with respect to the management style (see Figure 12). Its effect decays rather quickly and disappears after about 12 months. The results of these stress tests are compatible with the liquidity interpretation of the unobservable factor. We observe an immediate effect of the shock on the very liquid strategies of the Long/Short Equity Hedge management style, whereas the effect is clearly lagged, and indirectly due to contagion, for the Fixed Income Arbitrage strategies. The magnitude of the impact on the term structure depends on the conditioning information. There can be months when the sensitivity to shocks are larger, depending on the lagged liquidation counts at the month of the stress. In stress scenario S.2, the change in the contagion matrix is a permanent shock. In Figure 13, there is no important effect in the short run, but the long run behaviors of the models with and without the shock in the contagion matrix significantly differ for all styles, except for Global Macro. Indeed, the elements in the row of the estimated contagion matrix (Table 6) for that management style are zero. We conclude that there is no contagion effect impacting the Global Macro style. Therefore, the stress in scenario S.2 is irrelevant for the distribution of liquidation counts in that management style. Finally, when the frailty persistence parameter is shocked in stress scenario S.3, we observe an increase in the time at which the long run expected values of the liquidation counts are attained (Figure 14).

\section{Concluding remarks}

In this paper we develop a new methodology to analyse the dynamics of liquidation risk dependence in the hedge fund industry. The autoregressive Poisson model with dynamic frailty is especially appealing, since it allows for distinguishing the effect of exogenous shocks (L-effects) and the endogenous 
contagion effects (A-effects). We estimate this model by an appropriate method of moments, identify the channels through which the exogenous factor has an impact, and introduce different diagnostic tools. Furthermore, we explain how the model can be used for stress testing. The frailty and contagion components can also be disentangled in a more general (parametric) dynamic framework. The model can include more than one factor, lagged effects of these factors on the liquidation intensity, and an autoregressive order larger than one. The relative magnitude of the frailty and contagion components will depend on the selected number of factors and lags.

In addition to contagion and exogenous frailties shared by the entire population of HF, liquidation clustering can also be the consequence of type-specific effects. For instance, liquidation risk dependence can be due to sharing the same management company [Gregoriou, Pascalau (2012)] or part of the clients [Ben-David, Franzoni, Moussawi (2010)], or to being located in the same geographic area. In the latter case, the dependence can result from common clients, if there is a home bias effect of the investors [Sialm, Sun and Zheng (2012)]. Such liquidation risk dependence can be accommodated by refining the definition of type $k$, and including type-specific exogenous frailties $F_{k, t}$.

It is known that systemic risk can be due to a significant shock on a common factor amplified by contagion. Models with both frailty and contagion are required to understand what is the main channel of systemic risk, namely the exogenous shock and/or contagion, and to develop accurate strategies to avoid systemic crises. Concerning hedge funds, such an analysis would have to be performed jointly for market risk, that is on returns, and liquidation risk, that is on lifetimes. In this respect, our work completes the systemic risk analysis based on returns developed in a series of recent papers including Sadka (2010), Boyson, Stahel, Stulz (2010), Akay, Seniuz, Yoldas (2011), Brown et al. (2011), Billio et al. (2012), Bali, Brown, Caglayan (2012), and the analysis of comovements between inflows and outflows [Sialm, Sun and Zheng (2012)]. Moreover, liquidation can bias the performance analysis of hedge funds, as it is often a consequence of unexpected bad results. There exist an exten- 
sive literature on correcting the survivorship bias in individual mutual fund or HF performance [see e.g. Brown, Goetzmann, Ibbotson, Ross (1992), Carpenter, Lynch (1999) for mutual funds, and Fung, Hsieh (1997), (2000), Ackermann, McEnally, Ravenscraft (1999), Liang (2000), Baquero, Horst, Verbeek (2005) for hedge funds]. Liquidation risk dependence implies that the correction for survivorship bias should be performed jointly for all funds, and not individually fund by fund. The specification of a joint model for hedge fund returns and endogenous liquidation is an interesting avenue for future research. 


\section{References}

Acharya, V., Pedersen, L., Philippon, T., and M., Richardson (2010): "Measuring Systemic Risk", Working Paper, New York University.

Ackermann, C., McEnally, R., and D., Ravenscraft (1999): "The Performance of Hedge Funds: Risk, Return and Incentives", Journal of Finance, 54, 833-874.

Adrian, T., and M., Brunnermeier (2011): "CoVaR", Working Paper, Princeton University and Federal Reserve Bank of New York.

Ait-Sahalia, Y., Cacho-Diaz, J., and R., Laeven (2010): "Modeling Financial Contagion Using Mutually Exciting Jump Processes", Princeton Univ. DP.

Akay, O., Senyuz, Z., and E., Yoldas (2011): "Understanding Hedge Fund Contagion: A Markovswitching Dynamic Factor Approach", DP Bentley University.

Allen, F., and D., Gale (2000): "Financial Contagion", Journal of Political Economy, 108, 1-11.

Andrews, D., and W., Ploberger (1994): ”Optimal Tests When a Nuisance Parameter is Present Only Under the Alternative", Econometrica, 62, 1383-1414.

Azizpour, S., and K., Giesecke (2008): "Self-Exciting Corporate Defaults: Contagion vs Frailty", DP Stanford Univ.

Baba, N., and H., Goko (2006): "Survival Analysis of Hedge Funds", Bank of Japan, DP, 06-E-05.

Bali, T., Brown, S., and M., Caglayan (2012): "Systemic Risk and the Cross-Section of Hedge Funds Returns", Journal of Financial Economics, forthcoming.

Baquero, G., Horst, J., and M., Verbeek (2005): "Survivor, Look-Ahead Bias and the Performance of Hedge Funds", Journal of Financial and Quantitative Analysis, 40, 493-518.

Bates, D. (2006): "Maximum Likelihood Estimation of Latent Affine Processes", Review of Financial Studies, 19, 909-965.

Ben-David, I., Franzoni, F., and R., Moussawi (2010): "Caught in the Web: Exploring the Channels of Hedge Fund Contagion", DP Wharton School.

Billio, M., Getmansky, M., Lo, A., and L., Pellizzon (2012): ’Econometric Measures of Correlation and Systemic Risk in the Finance and Insurance Sectors", Journal of Financial Economics, 104, 535539.

Boyson, N. (2010): 'Implicit Incentives and Reputational Hedging by Hedge Fund Managers", Journal of Empirical Finance, 17, 283-299.

Boyson, N., Stahel, C., and R., Stulz (2010): "Hedge Fund Contagion and Liquidity Shocks”, Journal of Finance, 65, 1789-1816. 
Brown, S., Goetzmann, W., Ibbotson, R., and S., Ross (1992): "Survivor Bias in Performance Studies", Review of Financial Studies, 5, 553-580.

Brown, S., Goetzmann, W., and B., Liang (2004): "Fees on Fees in Funds of Funds", Journal of Investment Management, 3, 39-56.

Brown, S., Goetzmann, W., Liang, B., and C., Schwartz (2008): "Mandatory Disclosure and Operational Risk: Evidence From Hedge Fund Registration”, Journal of Finance, 63, 2785-2815.

Brown, S., Goetzmann, W., and J., Park (2001): "Careers and Survival Competition and Risks with Hedge Fund and CTA Industry", Journal of Finance, 56, 1869-1886.

Brown, S., Hwang, I., In, F., and T., Kim (2011): "Systemic Risk and Cross-Sectional Hedge Fund Returns", Working Paper.

Cameron, A., and P., Trivedi (1989): Regression Analysis of Count Data, Cambridge University Press, Cambridge.

Cappé, O., Moulines, E., and T., Rydén (2005): Inference in Hidden Markov Models, Springer Verlag. Carlson, M., and J., Steinman (2008): "Market Conditions and Hedge Fund Survival”, Federal Reserve Board, Washington, FEDS Working Paper 2008-28.

Carpenter, J., and A., Lynch (1999): "Survivorship Bias and Attrition Effects in Measures of Performance Persistence", Journal of Financial Economics, 54, 337-374.

Carrasco, M., Chernov, M., Florens, J.-P., and E., Ghysels (2007): "Efficient Estimation of General Dynamic Models with a Continuum of Moment Conditions", Journal of Econometrics, 140, 529-573.

Casey, Quirk, and Acito (2004): "Institutional Demand for Hedge Funds: New Opportunities and Standards", White Paper, CQA and the Bank of New-York, available on www.cqalle.com.

Chan, N., Getmansky, M., Haas, S. and A., Lo (2007): "Systemic Risk and Hedge Funds", in The Risks of Financial Institutions, ed. by M., Carey and R., Stulz, University of Chicago Press, 235-338.

Chesher, A. (1984): "Testing for Neglected Heterogeneity", Econometrica, 52, 865-872.

Cox, D. (1972): "Regression Models and Life Tables", Journal of the Royal Statistical Society, Series B, 34, 187-220.

Cox, D. (1983): "Some Remarks on Overdispersion", Biometrika, 70, 269-274.

Cox, J., Ingersoll, J., and S. Ross (1985): "A Theory of the Term Structure of Interest Rates", Econometrica, 53, 385-407.

Czado, C., Delwarde, A., and M., Denuit (2005): "Bayesian Poisson Log-bilinear Mortality Projections", Insurance: Mathematics and Economics, 36, 260-284.

Darolles, S., and C., Gourieroux (2012): "The Effects of Management and Provision Accounts on Hedge Fund Returns", CREST DP. 
Darolles, S., Gourieroux, C., and J., Jasiak (2006): "Structural Laplace Transform and Compound Autoregressive Models", Journal of Time Series Analysis, 27, 477-503.

Das, S., Duffie, D., Kapadia, N., and L., Saita (2007): "Common Failings: How Corporate Defaults are Correlated", Journal of Finance, 62, 93-117.

Duffie, D., Eckner, A., Horel, G., and L., Saita (2009): "Frailty Correlated Default", Journal of Finance, 64, 2089-2183.

Duffie, D., Filipovic, D., and W., Schachermayer (2003): "Affine Processes and Applications in Finance", Annals of Applied Probability, 13, 984-1053.

Dungey, M., Fry, R., Gonzalez-Hermosillo, B., and V., Martin (2010): Transmission of Financial Crises and Contagion: A Latent Factor Approach, Oxford University Press.

Feffer, S., and C., Kundro (2003): Understanding and Mitigating Operational Risk in Hedge Fund Investments, DP The Capital Markets Company Ltd.

Fung, W., and D., Hsieh (1997): "Investment Style and Survivorship Bias in the Returns of CTAs: The Information Content of Track Records", Journal of Portfolio Management, 24, 291-307.

Fung, W., and D., Hsieh (2000): "Performance Characteristics of Hedge Funds and Commodity Funds: Natural Versus Spurious Biases”, Journal of Financial and Quantitative Analysis, 35, 291-307.

Gagliardini, P., and C., Gourieroux (2011): ”Correlated Risks vs Contagion in Stochastic Transition Models", DP CREST.

Gagliardini, P., and C., Gourieroux (2012): "Identification by Laplace Transforms in Panel and Time Series Models with Unobserved Stochastic Time Effects”, DP CREST.

Getmansky, M. (2010): "The Life Cycle of Hedge Funds: Fund Flows, Size and Performance”, DP MIT.

Getmansky, M., Lo, A., and S., Mei (2004): "Sifting Through the Wreckage: Lessons from Recent Hedge-Fund Liquidations", Journal of Investment Management, 2, 6-38.

Giesecke, K., and S., Weber (2004): "Cyclical Correlations, Credit Contagion and Portfolio Losses", Journal of Banking and Finance, 28, 3009-3036.

Gourieroux, C., and J., Jasiak (2006): ”Autoregressive Gamma Process”, Journal of Forecasting, 25, 129-152.

Gregoriou, G., Lhabitant, F.-S., and F., Rouah (2010): "Survival of Exchange-Listed Hedge Funds", Journal of Applied Research in Accounting and Finance, 4, 2-11.

Gregoriou, G., and R., Pascalau (2012): "Joint Survival Analysis of Hedge Funds and Funds of Funds Using Copulas"”, Managerial Finance, 38, 82-100. 
Hawkes, A. (1971): "Point Spectra of Some Mutually Exciting Point Processes", Journal of the Royal Statistical Society, Series B, 33, 438-443.

Hawkes, A., and D., Oakes (1974): "A Cluster Representation of a Self-Exciting Process”, Journal of Applied Probability, 11, 493-503.

Lando, D., and M., Nielsen (2010): "Correlation in Corporate Defaults: Contagion or Conditional Independence?", Journal of Financial Intermediation, 19, 355-372.

Liang, B. (2000): "Hedge Funds: The Living and the Dead", Journal of Financial and Quantitative Analysis, 35, 309-326.

Malkiel, B., and A., Saha (2005): "Hedge Funds: Risk and Return”, Financial Analysts Journal, 61, 80-88.

Manski, C. (1993): "Identification of Endogenous Social Effects: The Reflection Problem", Review of Economic Studies, 60, 531-542.

McCullagh, P., and J., Nelder (1989): Generalized Linear Models, 2nd Edition, Chapman Hall.

Oakes, D. (1989): "Bivariate Survival Models Induced by Frailties", Journal of the American Statistical Society, 84, 487-493.

Sadka, R. (2010): 'Liquidity Risk and the Cross Section of Hedge Fund Returns”, Journal of Financial Economics, 98, 54-71.

Sialm, C., Sun, Z., and L., Zheng (2012): "Home Bias and Local Contagion: Evidence From Funds of Funds", Working Paper, University of California Irvine.

Vaupel, J., Manton, K., and E., Stallard (1979): "The Impact of Heterogeneity in Individual Frailty on the Dynamics of Mortality", Demography, 16, 439-454.

White, H. (1982): "Maximum Likelihood Estimation of Misspecified Models", Econometrica, 50, $1-25$. 
Figure 1: Subpopulation sizes of HF.
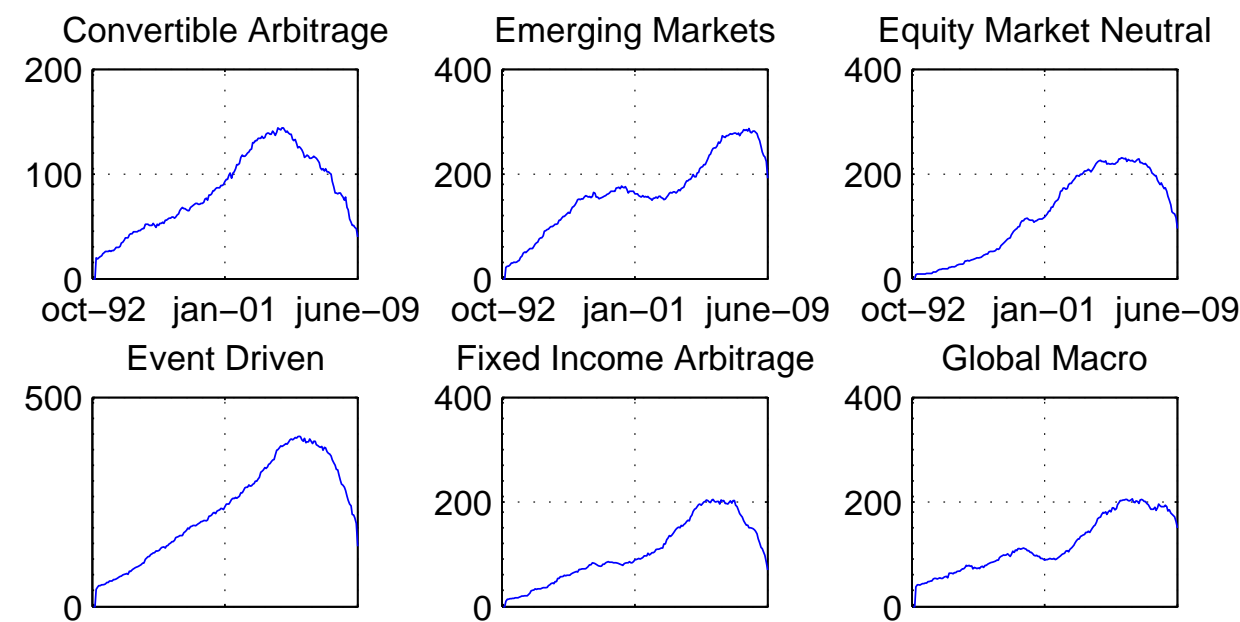

oct-92 jan-01 june-09

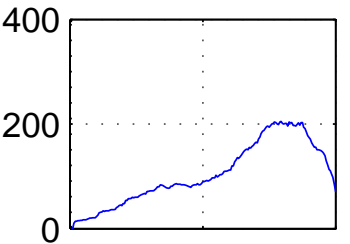

Global Macro

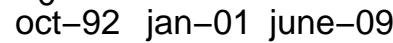

oct-92 jan-01 june-09

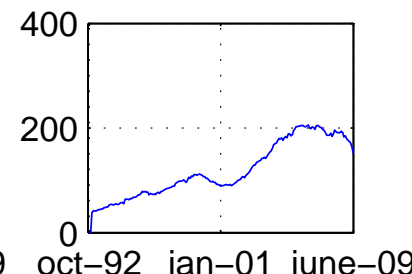

Long/Short Equity Hedge

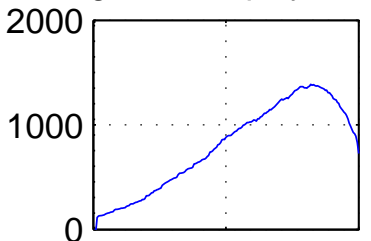

Managed Futures

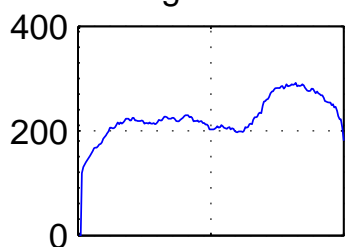

Multi-Strategy

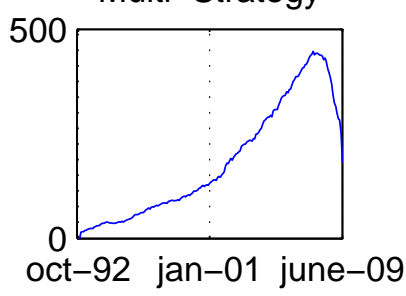

The figure displays the evolution of the population size $n_{k, t}$ between October 1992 and June 2009 for the nine management styles. Data are aggregated w.r.t. age. 
Figure 2: Liquidation rates of HF.

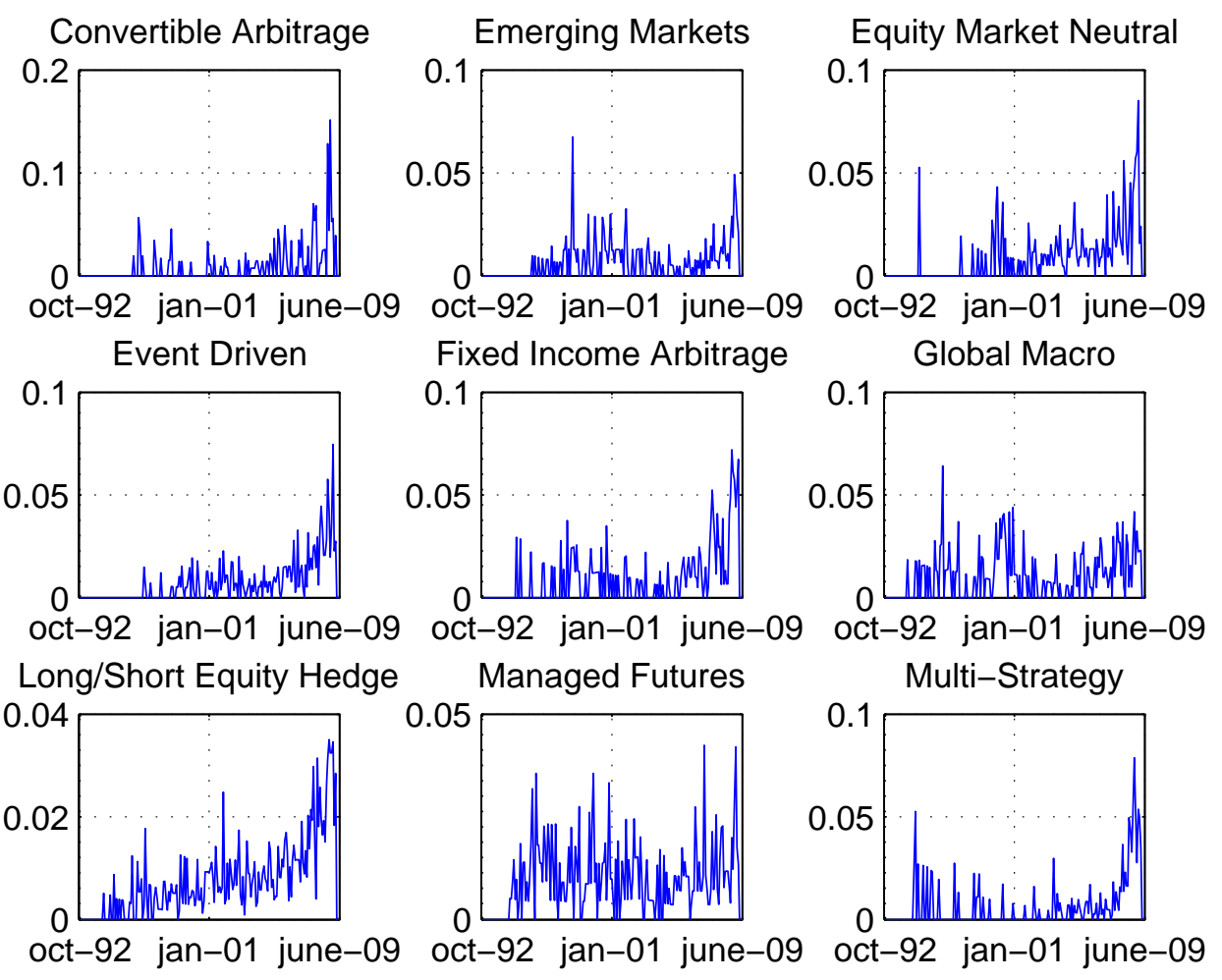

The figure displays the time series of liquidation rate $Y_{k, t} / n_{k, t}$ between October 1992 and June 2009 for the nine management styles. Data are aggregated w.r.t. age. 
Figure 3: Smoothed estimates of liquidation intensity.
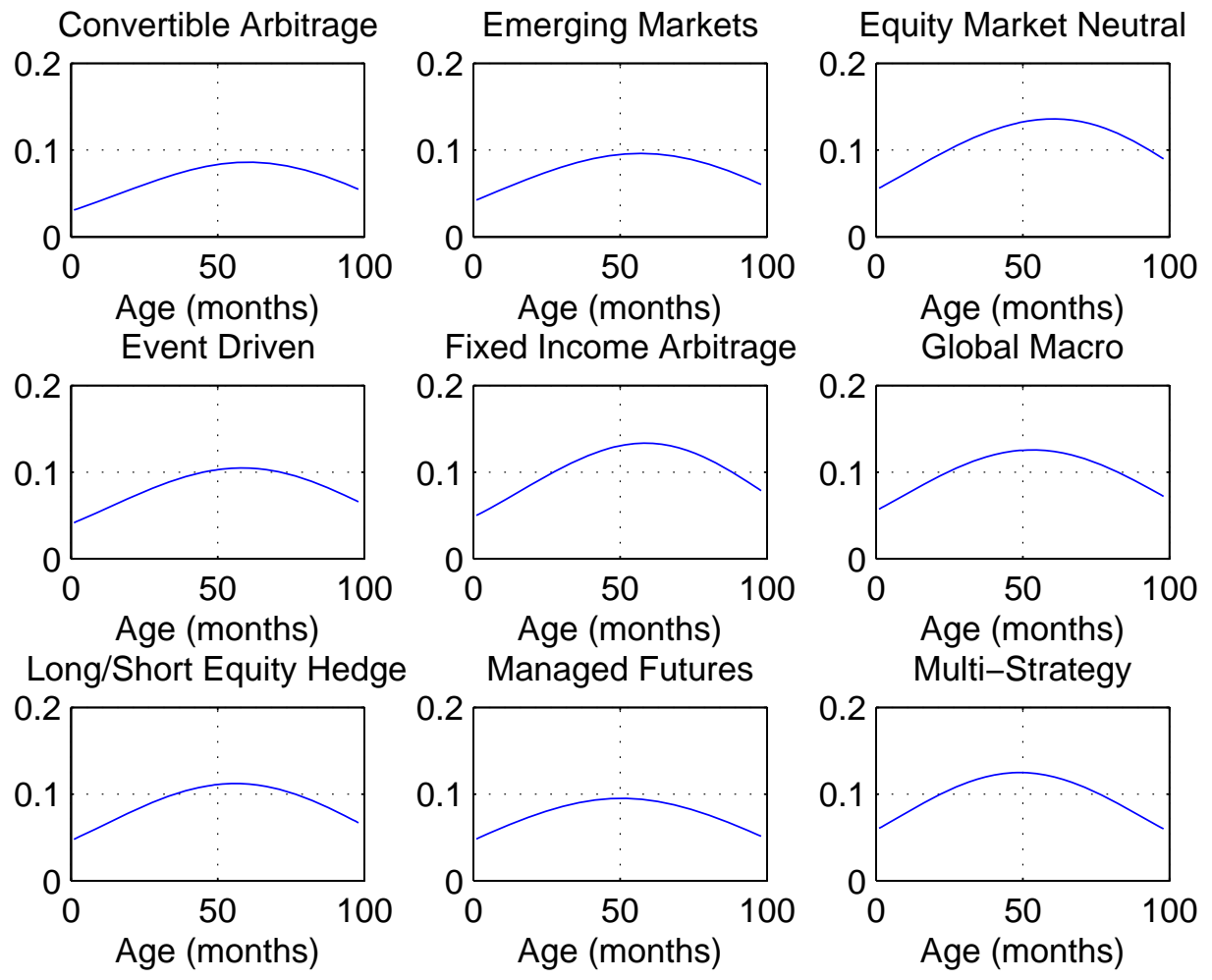

The figure displays the smoothed nonparametric estimates of the liquidation intensity as a function of age for the nine management styles. The estimates are based on the Kaplan-Meier estimators of the historical survival functions. 
Figure 4: Lexis diagram.

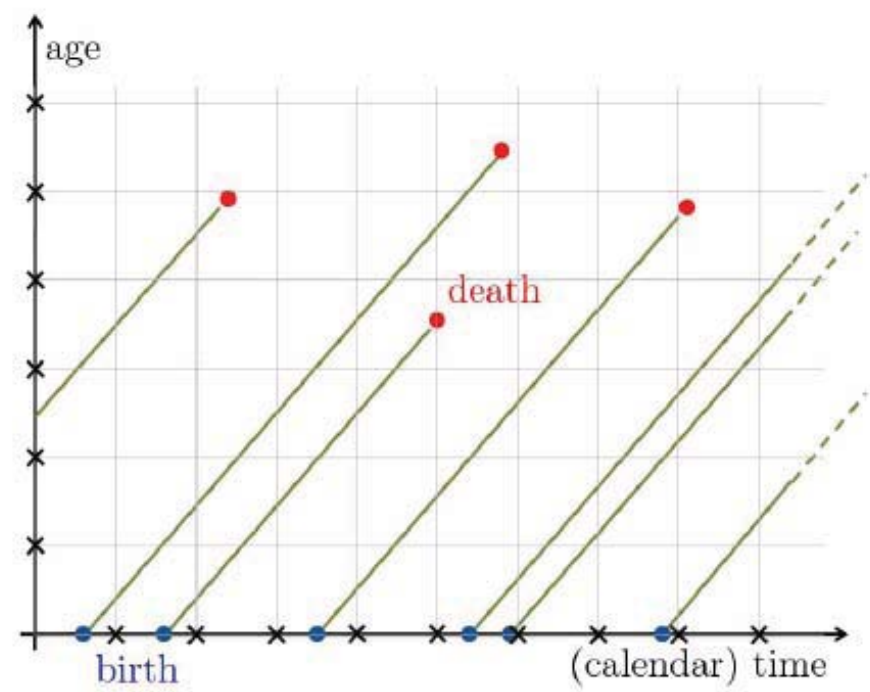

In the lexis diagram, the liquidation of a HF is represented by a dot in the plane. The horizontal axis corresponds to the calendar time of the liquidation event, while the vertical axis displays the age of the fund at liquidation. The diagonal 45-degree lines correspond to funds in a same cohort. 
Figure 5: Lexis diagram for Emerging Markets.

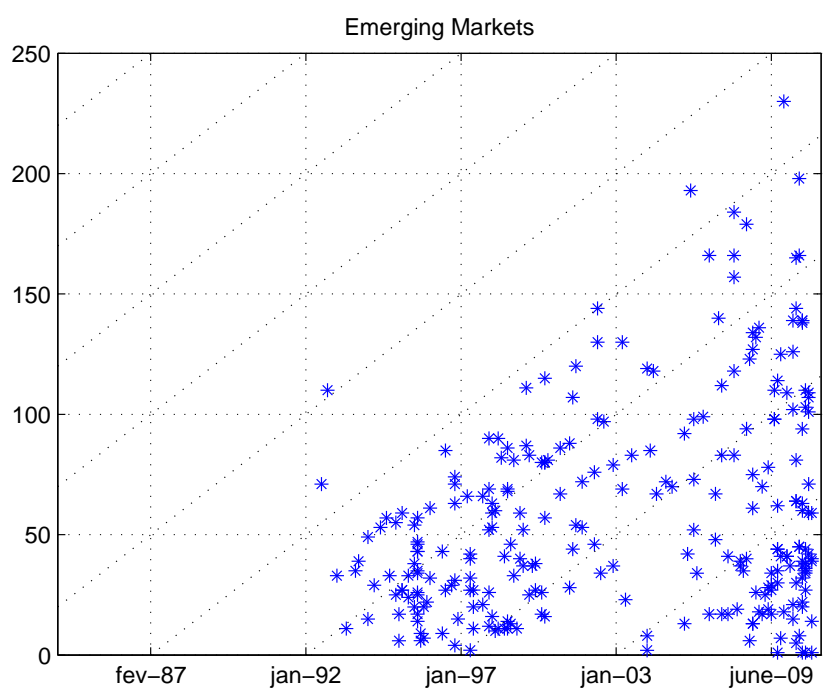

The figure displays the lexis diagram for liquidation events of HF with management style Emerging Markets in the TASS database. The horizontal axis represents calendar time and the vertical axis represents age in months.

Figure 6: Lexis diagram for Global Macro.

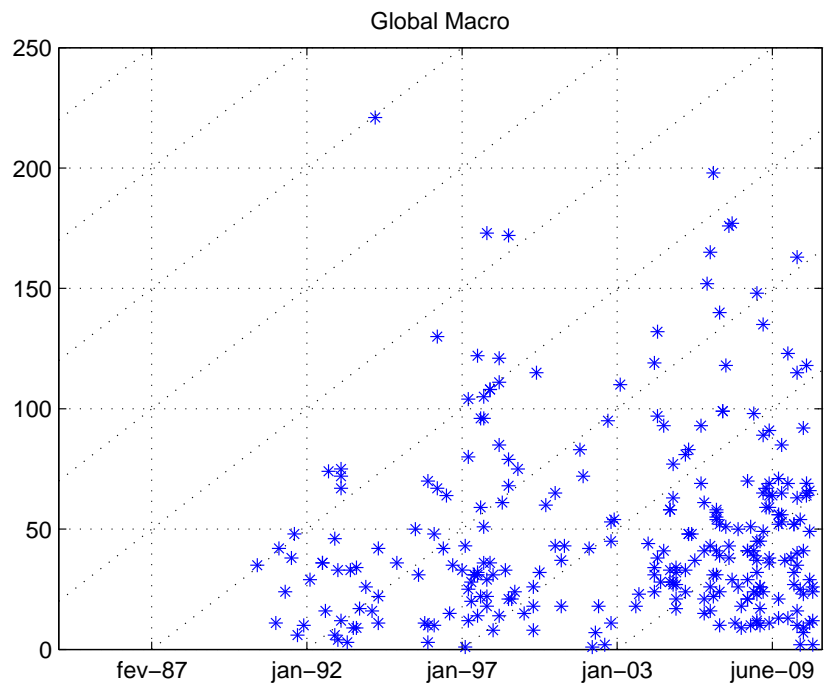

The figure displays the lexis diagram for liquidation events of HF with management style Global Macro in the TASS database. The horizontal axis represents calendar time and the vertical axis represents age in months. 
Figure 7: Lexis diagram for Multi Strategy.

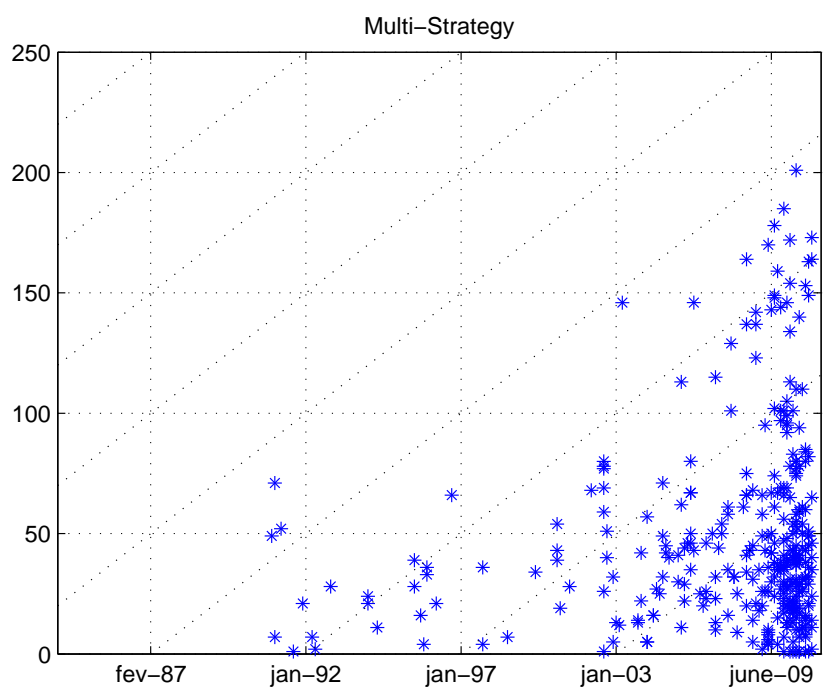

The figure displays the lexis diagram for liquidation events of HF with management style Multi Strategy in the TASS database. The horizontal axis represents calendar time and the vertical axis represents age in months.

Figure 8: Lexis diagram for Managed Futures.

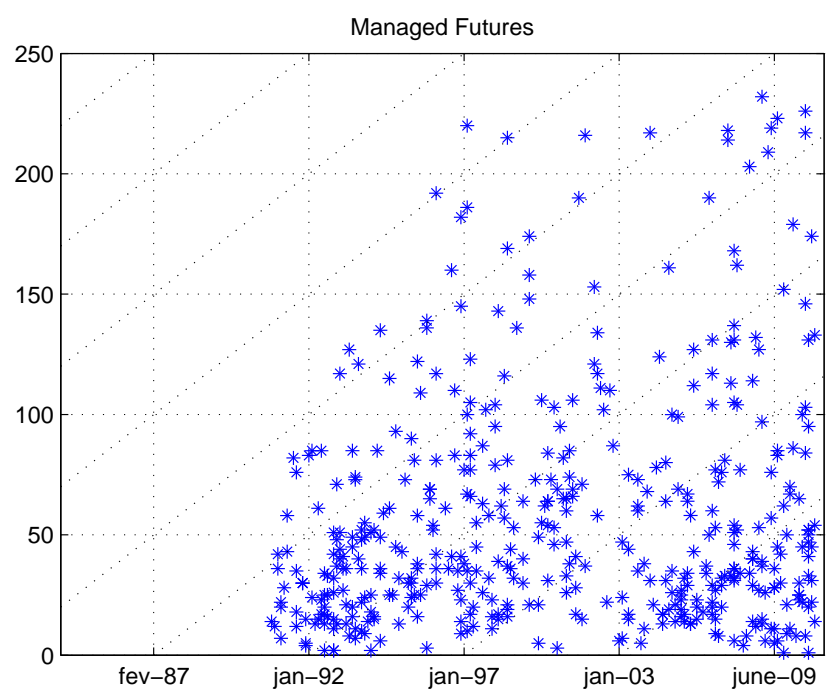

The figure displays the lexis diagram for liquidation events of HF with management style Managed Futures in the TASS database. The horizontal axis represents calendar time and the vertical axis represents age in months. 
Figure 9: The contagion scheme for the pure contagion model.

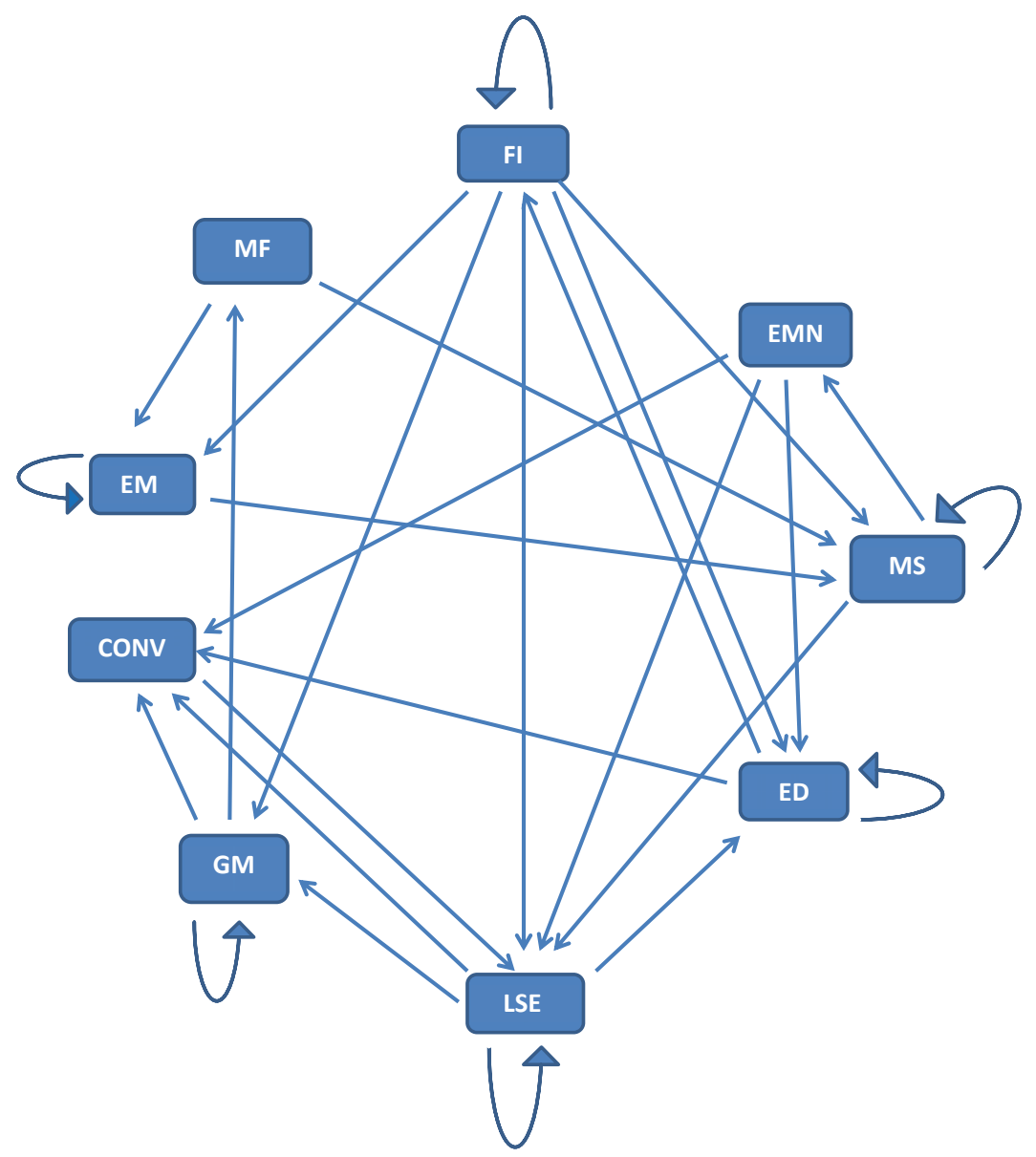

The figure provides the contagion scheme for the Poisson model with contagion only estimated on the TASS database. We display an arrow between two management styles, if the estimated contagion coefficient from the first style to the second style is statistically significant at $5 \%$ level. The estimated contagion matrix is provided in Table 4. 
Figure 10: The contagion scheme for the model with contagion and frailty.

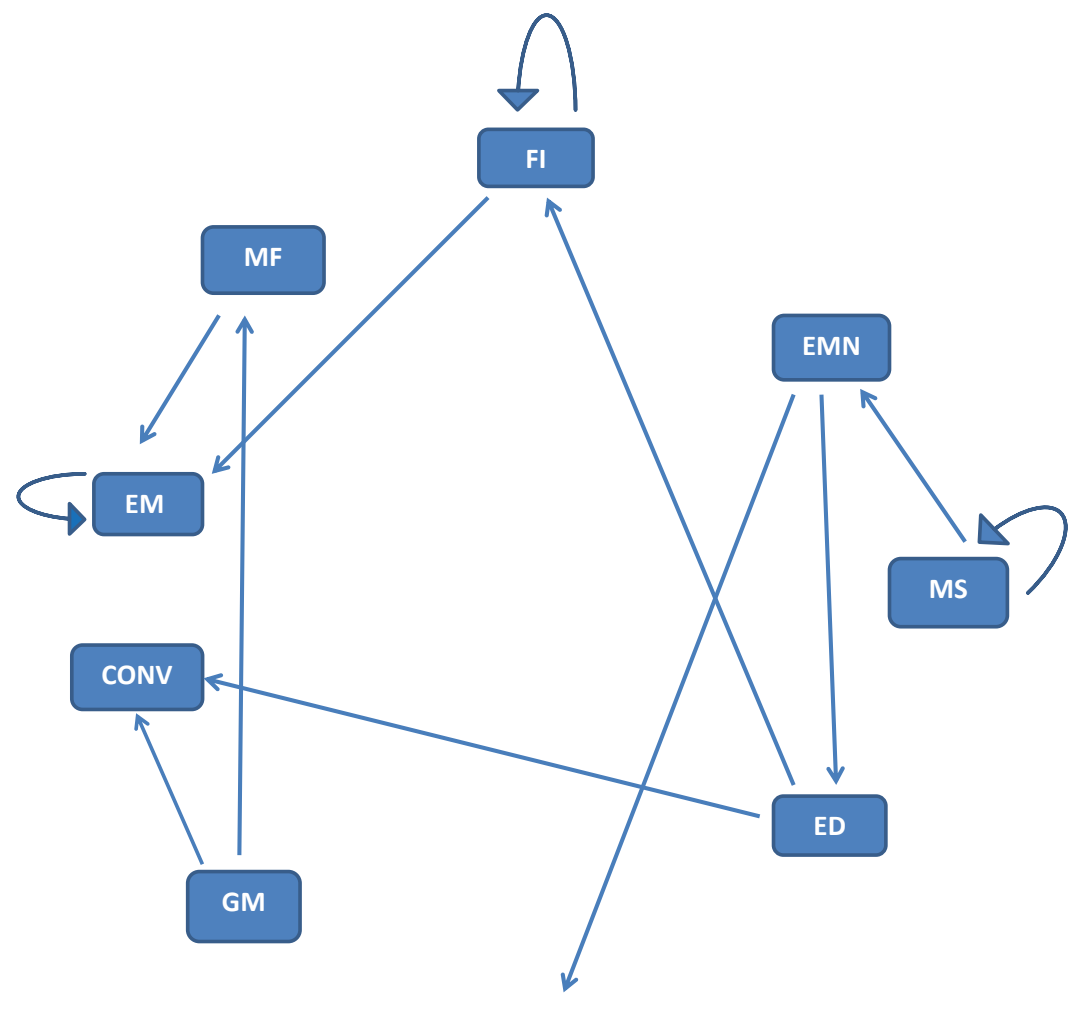

LSE

The figure provides the contagion scheme for the Poisson model with both frailty and contagion estimated on the TASS database. We display an arrow between two management styles, if the estimated contagion coefficient from the first style to the second style is statistically significant at $5 \%$ level. The estimated contagion matrix is provided in Table 6. 
Figure 11: (Cross-)ACF of residuals for diagnostic check.
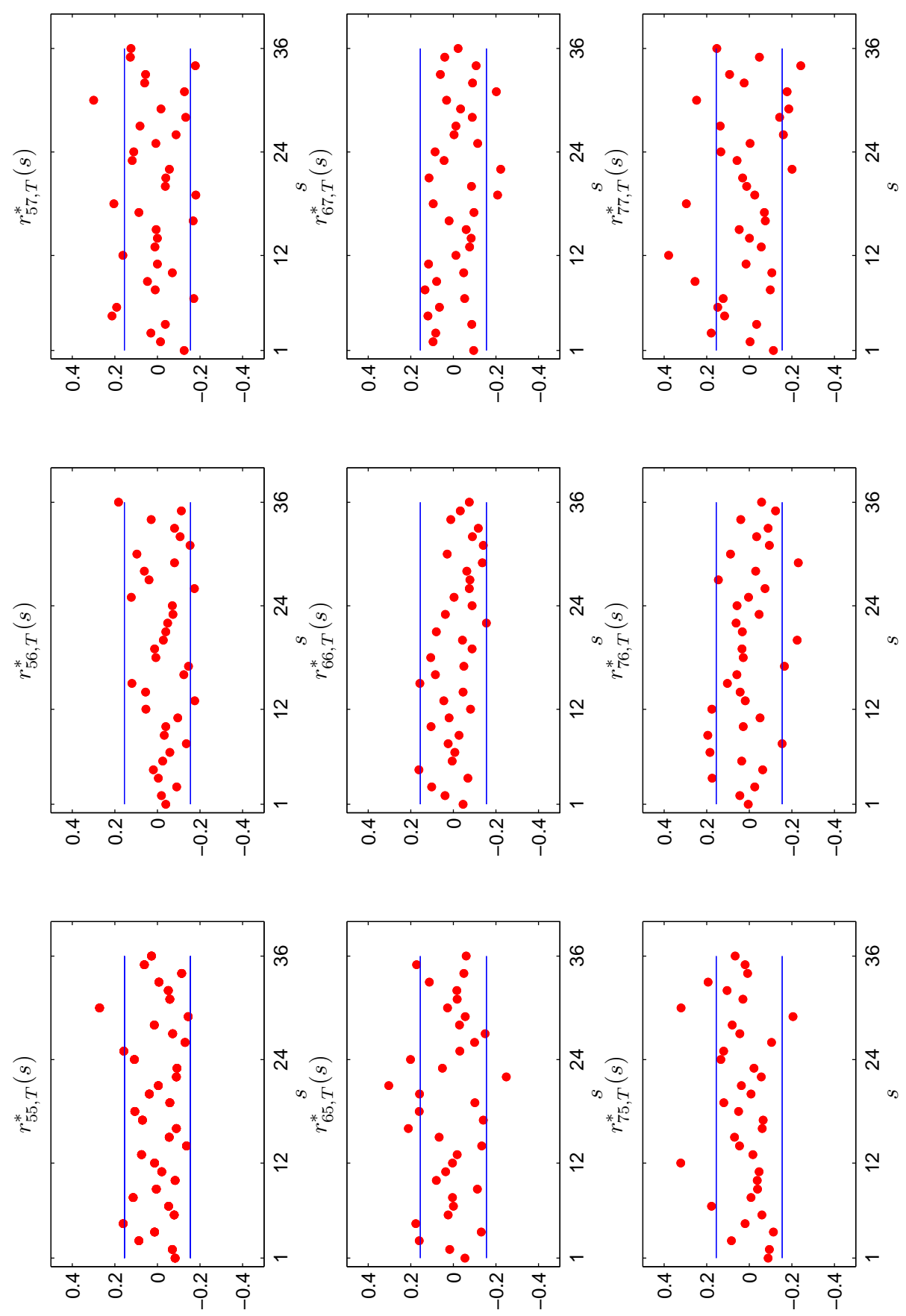

The figure displays the auto- and cross-correlation functions of residuals $r_{k l, T}^{*}(s)$, with $s$ varying, defined in equation (3.13), for the management styles Fixed Income Arbitrage $(k=5)$, Global Macro $(k=6)$ and Long/Short Equity Hedge $(k=7)$. The horizontal lines are $95 \%$ confidence bands. 
Figure 12: Term structure of expected liquidation counts when stressing the current factor value.
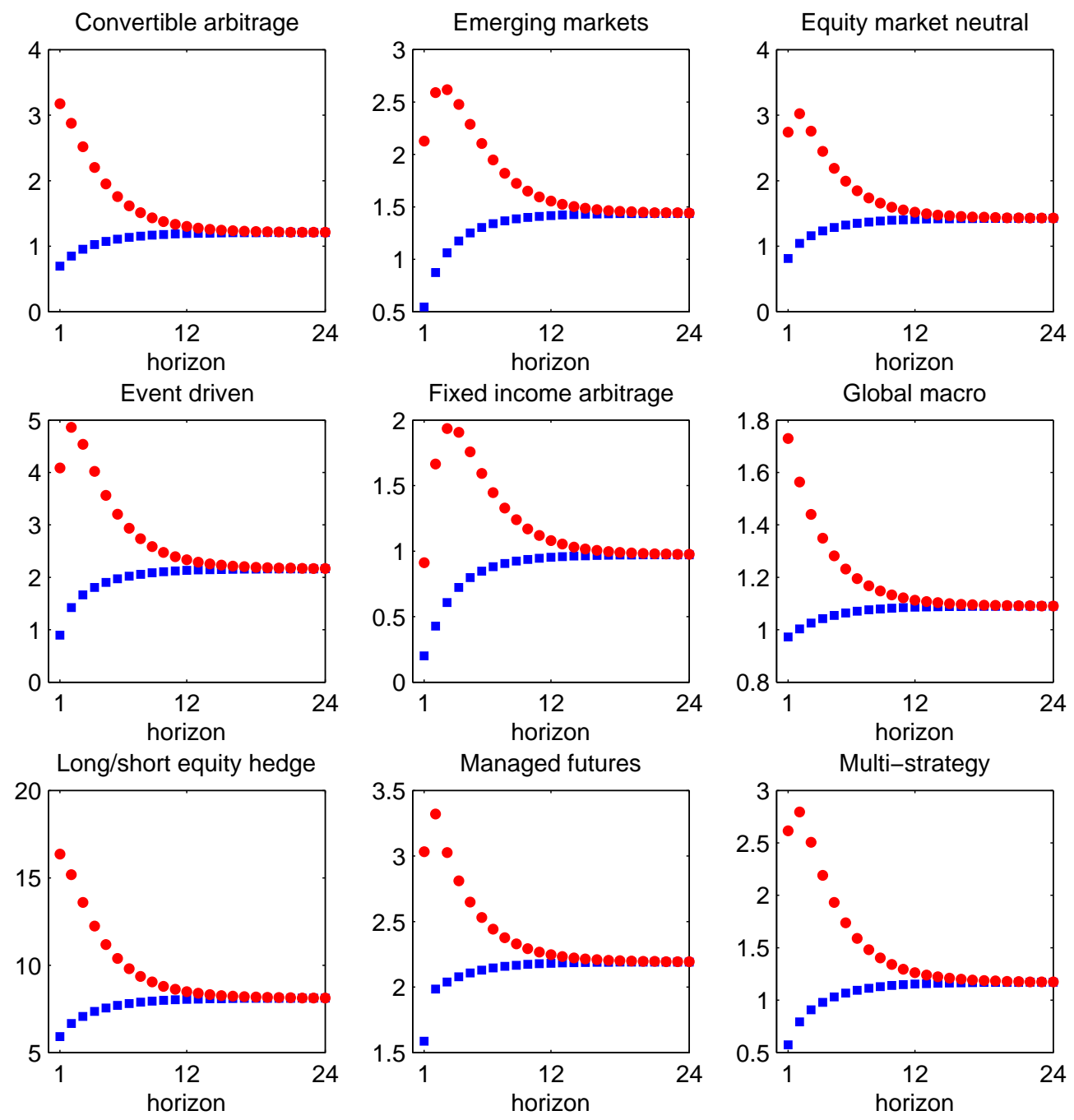

Term structure of the conditional expectation $E\left[Y_{k, t+\tau} \mid Y_{t}, F_{t}\right]$ of liquidation counts for horizon $\tau=$ $1,2, \ldots, 24$ months, by management style $k$. Squares and circles correspond to conditioning sets with $F_{t}$ equal to the median and the $95 \%$ quantile, respectively, of the stationary distribution of the frailty. The liquidation counts vector $Y_{t}$ in the conditioning set corresponds to the observations in June 2009 for both curves. The model is the specification including frailty and contagion, with intensity parameters as in Tables 5 and 6 , and frailty dynamic parameters $\delta=0.59$ and $\rho=0.74$, corresponding to the estimates of Section 3.4. 
Figure 13: Term structure of expected liquidation counts when stressing the contagion matrix.
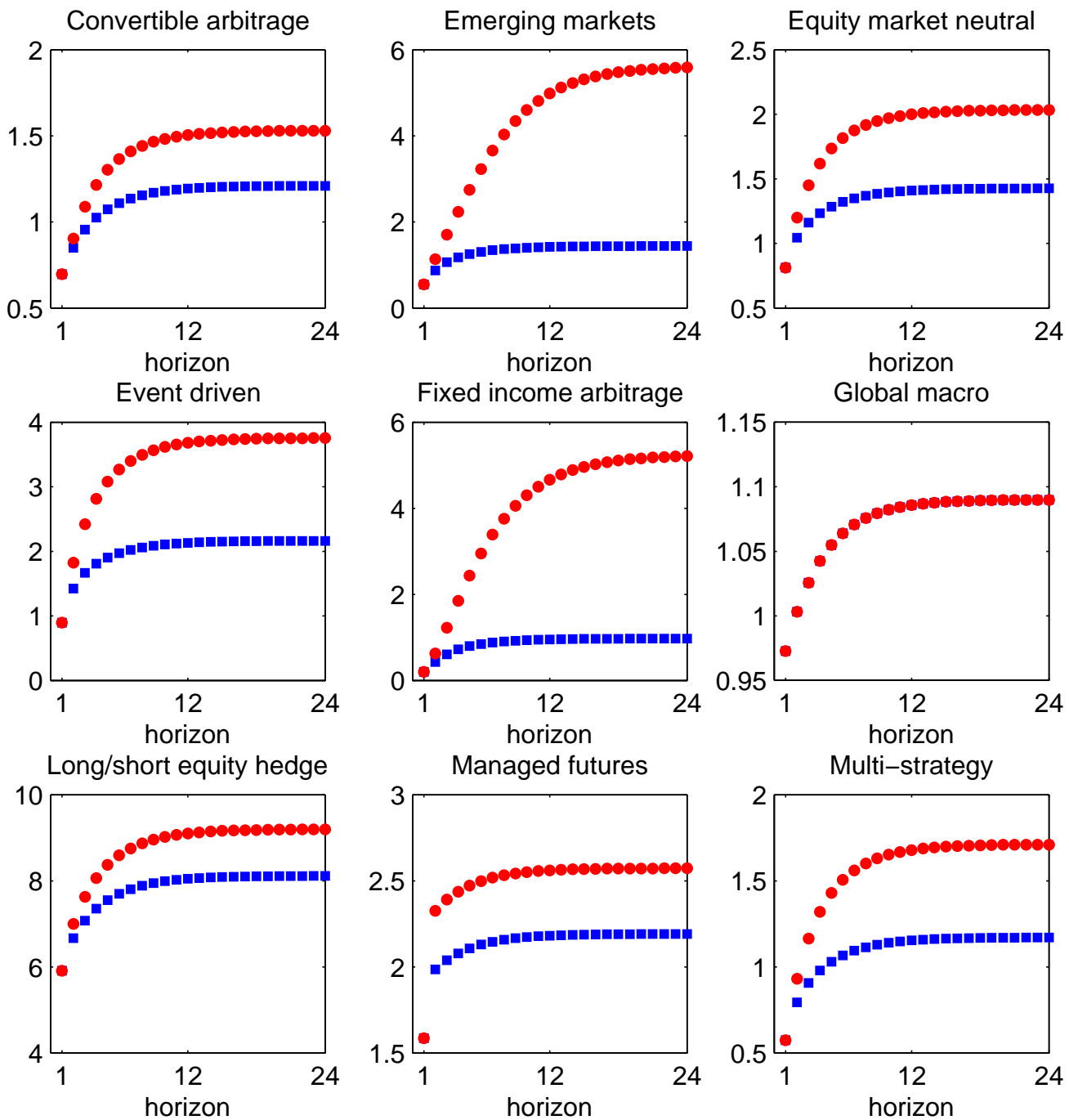

Term structure of the conditional expectation $E\left[Y_{k, t+\tau} \mid Y_{t}, F_{t}\right]$ of liquidation counts for horizon $\tau=$ $1,2, \ldots, 24$ months, by management style $k$. Squares and circles correspond to models with contagion matrices $C^{s}=\hat{C}$ and $C^{s}=2 \hat{C}$, respectively, where $\hat{C}$ is the matrix of estimates in Table 6 . The intercepts and frailty sensitivities are as in Table 5 , and the frailty dynamic parameters are $\delta=0.59$ and $\rho=0.74$, corresponding to the estimates of Section 3.4. The factor value $F_{t}$ in the conditioning set corresponds to the median of the stationary distribution of the frailty, while the liquidation counts vector $Y_{t}$ in the conditioning set corresponds to the observations in June 2009 for both curves. 
Figure 14: Term structure of expected liquidation counts when stressing the frailty persistence.
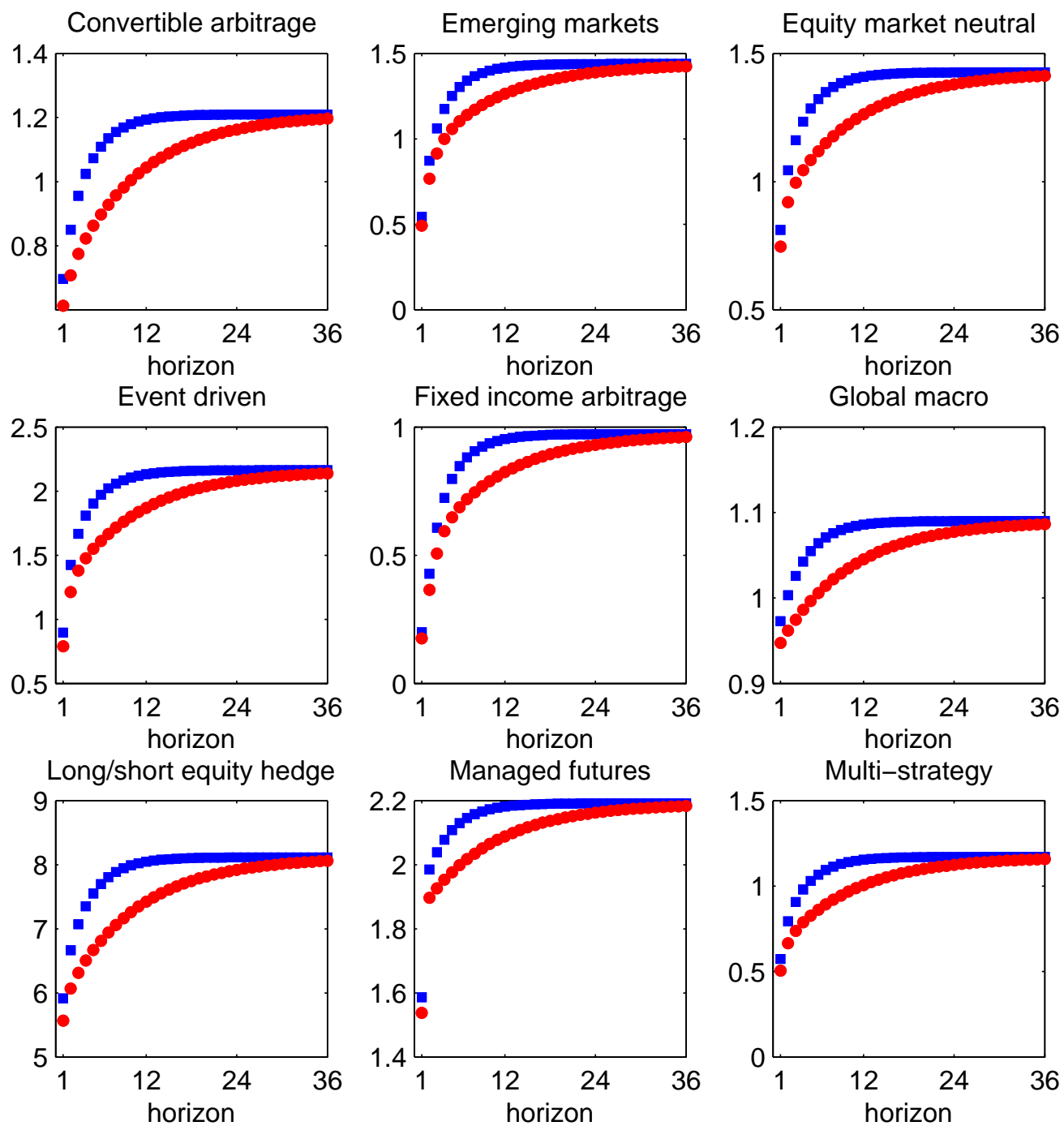

Term structure of the conditional expectation $E\left[Y_{k, t+\tau} \mid Y_{t}, F_{t}\right]$ of liquidation counts for horizon $\tau=$ $1,2, \ldots, 24$ months, by management style $k$. Squares and circles correspond to models with frailty autocorrelation $\rho^{s}=0.74$ (corresponding to the estimate in Section 3.4) and $\rho^{s}=0.90$, respectively. The intensity parameters are as in Tables 5 and 6 , and the parameter characterizing the stationary distribution of the frailty is $\delta=0.59$, corresponding to the estimate of Section 3.4. The factor value $F_{t}$ in the conditioning set corresponds to the median of the stationary distribution of the frailty, while the liquidation counts vector $Y_{t}$ in the conditioning set corresponds to the observations in June 2009 for both curves. 
Table 1: The database.

\begin{tabular}{ll|cccc|cc}
\hline \hline & & & & & & \\
& & Alive funds & $(\%)$ & Liquidated funds & $(\%)$ & Total & $(\%)$ \\
\hline & & & & & & & \\
CONV & Convertible Arbitrage & 45 & $2.00 \%$ & 66 & $4.30 \%$ & $\mathbf{1 1 1}$ & $2.90 \%$ \\
EM & Emerging Markets & 227 & $10.00 \%$ & 11 & $7.30 \%$ & $\mathbf{3 3 8}$ & $8.90 \%$ \\
EMN & Equity Market Neutral & 126 & $5.50 \%$ & 139 & $9.10 \%$ & $\mathbf{2 6 5}$ & $7.00 \%$ \\
ED & Event Driven & 216 & $9.50 \%$ & 129 & $8.50 \%$ & $\mathbf{3 4 5}$ & $9.10 \%$ \\
FI & Fixed Income Arbitrage & 95 & $4.20 \%$ & 75 & $4.90 \%$ & $\mathbf{1 7 0}$ & $4.50 \%$ \\
GM & Global Macro & 162 & $7.10 \%$ & 102 & $6.70 \%$ & $\mathbf{2 6 4}$ & $6.90 \%$ \\
LSE & Long/Short Equity Hedge & 885 & $38.80 \%$ & 546 & $35.90 \%$ & $\mathbf{1 4 3 1}$ & $37.70 \%$ \\
MF & Managed Futures & 224 & $9.80 \%$ & 230 & $15.10 \%$ & $\mathbf{4 5 4}$ & $12.00 \%$ \\
MS & Multi-Strategy & 299 & $13.10 \%$ & 122 & $8.00 \%$ & $\mathbf{4 2 1}$ & $11.10 \%$ \\
& & & & & & & $\mathbf{1 0 0 . 0 0 \%}$ \\
\hline
\end{tabular}

The table provides the distribution of alive and liquidated funds across the nine management styles in June 2009. 
Table 2: Maximum and boundary values of the liquidation rates.

\begin{tabular}{l|ccc}
\hline \hline & & & \\
& Lower Boundary & Maximum & Upper Boundary \\
\hline & & & \\
Convertible Arbitrage & 0.022 & 0.075 & 0.051 \\
Emerging Markets & 0.034 & 0.082 & 0.051 \\
Equity Market Neutral & 0.045 & 0.124 & 0.084 \\
Event Driven & 0.032 & 0.093 & 0.058 \\
Fixed Income Arbitrage & 0.040 & 0.114 & 0.066 \\
Global Macro & 0.045 & 0.108 & 0.066 \\
Long/Short Equity Hedge & 0.038 & 0.102 & 0.062 \\
Managed Futures & 0.041 & 0.086 & 0.045 \\
Multi-Strategy & 0.052 & 0.119 & 0.058 \\
& & & \\
\hline \hline
\end{tabular}

The table provides the value of the estimated liquidation intensity at the lower and upper bounderies of the age domain, as well as the maximum estimated value of the liquidation intensity. The lower and upper boundaries of the age domain are 0 months and 100 months from inception, respectively. 
Table 3: Estimated intercepts in the pure contagion model.

\begin{tabular}{l|c}
\hline \hline & Intercept $a_{k}$ \\
\hline \multirow{3}{*}{ Convertible Arbitrage } & 0.05 \\
Emerging Markets & $(0.11)$ \\
& $0.51^{* * *}$ \\
Equity Market Neutral & $(0.15)$ \\
& $0.63^{* * *}$ \\
Event Driven & $(0.17)$ \\
& $0.59^{* * *}$ \\
Fixed Income Arbitrage & $(0.21)$ \\
& $0.30^{* *}$ \\
Global Macro & $(0.13)$ \\
& $0.73^{* * *}$ \\
Long/Short Equity Hedge & $(0.13)$ \\
& $4.56^{* * *}$ \\
Managed Futures & $(0.46)$ \\
& $1.65^{* * *}$ \\
Multi-Strategy & $(0.22)$ \\
& $0.21^{* *}$ \\
\hline \hline
\end{tabular}

The table provides the Maximum Likelihood (ML) estimates of the intercept parameters $a_{k}$ for the Poisson model with contagion only. Standard errors are provided in parentheses. Stars $*, * *$ and $* * *$ denote significance at the $10 \%, 5 \%$ and $1 \%$ level, respectively. 
Table 4: Estimated contagion parameters in the pure contagion model.

\begin{tabular}{|c|c|c|c|c|c|c|c|c|c|}
\hline & $\begin{array}{l}\text { Convertible } \\
\text { Arbitrage }\end{array}$ & $\begin{array}{l}\text { Emerging } \\
\text { Markets }\end{array}$ & $\begin{array}{l}\text { Equity } \\
\text { Market } \\
\text { Neutral }\end{array}$ & $\begin{array}{l}\text { Event } \\
\text { Driven }\end{array}$ & $\begin{array}{c}\text { Fixed } \\
\text { Income } \\
\text { Arbitrage }\end{array}$ & $\begin{array}{l}\text { Global } \\
\text { Macro }\end{array}$ & $\begin{array}{l}\text { Long/Short } \\
\text { Equity Hedge }\end{array}$ & $\begin{array}{l}\text { Managed } \\
\text { Futures }\end{array}$ & $\begin{array}{l}\text { Multi- } \\
\text { Strategy }\end{array}$ \\
\hline Convertible Arbitrage & & & $\begin{array}{c}-0.13^{* *} \\
(0.06)\end{array}$ & $\begin{array}{l}0.11^{* *} \\
(0.05)\end{array}$ & & $\begin{array}{c}0.20^{* * *} \\
(0.07)\end{array}$ & $\begin{array}{r}0.05^{* *} \\
(0.02)\end{array}$ & & \\
\hline Emerging Markets & & $\begin{array}{c}0.19^{* * *} \\
(0.07)\end{array}$ & & & $\begin{array}{c}0.31^{* * *} \\
(0.09)\end{array}$ & & & $\begin{array}{c}0.17^{* * *} \\
(0.05)\end{array}$ & \\
\hline Equity Market Neutral & & & & & & & & & $\begin{array}{c}0.24^{* * *} \\
(0.09)\end{array}$ \\
\hline Event Driven & & & $\begin{array}{c}0.35^{* * *} \\
(0.11)\end{array}$ & $\begin{array}{l}0.16^{* *} \\
(0.07)\end{array}$ & $\begin{array}{c}0.36^{* * *} \\
(0.13)\end{array}$ & & $\begin{array}{c}0.08^{* * *} \\
(0.03)\end{array}$ & & \\
\hline Fixed Income Arbitrage & & & & $\begin{array}{c}0.08^{* *} \\
(0.04)\end{array}$ & $\begin{array}{c}0.32^{* * *} \\
(0.08)\end{array}$ & & & & \\
\hline Global Macro & & & & & $\begin{array}{c}0.20^{* * *} \\
(0.07)\end{array}$ & $\begin{array}{c}0.23^{* * *} \\
(0.08)\end{array}$ & $\begin{array}{c}-0.04^{* *} \\
(0.02)\end{array}$ & & \\
\hline Long/Short Equity Hedge & $\begin{array}{c}0.42^{* *} \\
(0.17)\end{array}$ & & $\begin{array}{c}0.42^{* *} \\
(0.20)\end{array}$ & & $\begin{array}{c}0.67^{* * *} \\
(0.25)\end{array}$ & & $\begin{array}{c}0.21^{* * *} \\
(0.06)\end{array}$ & & $\begin{array}{c}0.78^{* * *} \\
(0.23)\end{array}$ \\
\hline Managed Futures & & & & & & $\begin{array}{c}0.27^{* *} \\
(0.11)\end{array}$ & & & \\
\hline Multi-Strategy & & $\begin{array}{c}-0.16^{* * *} \\
(0.04)\end{array}$ & & & $\begin{array}{c}0.15^{* * *} \\
(0.06)\end{array}$ & & & $\begin{array}{c}-0.07^{* *} \\
(0.03)\end{array}$ & $\begin{array}{c}0.47^{* * *} \\
(0.07)\end{array}$ \\
\hline
\end{tabular}

The table provides the Maximum Likelihood (ML) estimates of the contagion parameters $\left(c_{k, k^{\prime}}\right)$ for the Poisson model with contagion only. Rows and columns correspond to target and source of contagion, respectively. Standard errors are provided in parentheses. Stars $* *$ and $* * *$ denote significance at the $5 \%$ and $1 \%$ level, respectively. The estimates that are not statistically significant at $5 \%$ level are not displayed. 
Table 5: Estimated intercepts and factor sensitivities in the model with contagion and frailty.

\begin{tabular}{l|cc}
\hline \hline & & \\
& Intercept $a_{k}$ & Sensitivity $b_{k}$ \\
\hline \multirow{3}{*}{ Convertible Arbitrage } & 0.00 & $1.08^{* *}$ \\
Emerging Markets & $(0.15)$ & $(0.55)$ \\
& 0.10 & $0.69^{* *}$ \\
Equity Market Neutral & $(0.23)$ & $(0.27)$ \\
& 0.27 & $0.84^{* *}$ \\
Event Driven & $(0.30)$ & $(0.40)$ \\
Fixed Income Arbitrage & 0.00 & $1.39^{* *}$ \\
& $(0.15)$ & $(0.70)$ \\
Global Macro & 0.00 & $0.31^{* *}$ \\
Long/Short Equity Hedge & $(0.20)$ & $(0.13)$ \\
& $0.76^{* * *}$ & $0.33^{* * *}$ \\
Managed Futures & $(0.14)$ & $(0.12)$ \\
& $2.98^{* * *}$ & $4.55^{* *}$ \\
Multi-Strategy & $(1.10)$ & $(1.92)$ \\
& 1.18 & $0.63^{* *}$ \\
\hline \hline
\end{tabular}

The table provides the estimates of the intercepts $a_{k}$ and frailty sensitivities $b_{k}$ for the Poisson model with frailty and contagion. The estimates are obtained by the Generalized Method of Moments (GMM) estimator of Section 3.4 iii). Standard errors are provided in parentheses. Stars $*$, $* *$ and $* * *$ denote significance at the $10 \%, 5 \%$ and $1 \%$ level, respectively. 
Table 6: Estimated contagion parameters in the model with contagion and frailty.

\begin{tabular}{|c|c|c|c|c|c|c|c|c|c|}
\hline & $\begin{array}{l}\text { Convertible } \\
\text { Arbitrage }\end{array}$ & $\begin{array}{l}\text { Emerging } \\
\text { Markets }\end{array}$ & $\begin{array}{l}\text { Equity } \\
\text { Market } \\
\text { Neutral }\end{array}$ & $\begin{array}{l}\text { Event } \\
\text { Driven }\end{array}$ & $\begin{array}{c}\text { Fixed } \\
\text { Income } \\
\text { Arbitrage }\end{array}$ & $\begin{array}{l}\text { Global } \\
\text { Macro }\end{array}$ & $\begin{array}{c}\text { Long/Short } \\
\text { Equity Hedge }\end{array}$ & $\begin{array}{l}\text { Managed } \\
\text { Futures }\end{array}$ & $\begin{array}{l}\text { Multi- } \\
\text { Strategy }\end{array}$ \\
\hline Convertible Arbitrage & & & & & & $\begin{array}{c}0.15^{* *} \\
(0.07)\end{array}$ & & & \\
\hline Emerging Markets & & $\begin{array}{c}0.17^{* * *} \\
(0.06)\end{array}$ & & & $\begin{array}{c}0.21^{* *} \\
(0.08)\end{array}$ & & & $\begin{array}{c}0.20^{* * *} \\
(0.05)\end{array}$ & \\
\hline Equity Market Neutral & & & & & & & & & $\begin{array}{l}0.10^{* *} \\
(0.05)\end{array}$ \\
\hline Event Driven & & & $\begin{array}{c}0.35^{* * *} \\
(0.09)\end{array}$ & $\begin{array}{l}0.10^{*} \\
(0.06)\end{array}$ & & & & & \\
\hline Fixed Income Arbitrage & & & & $\begin{array}{c}0.09^{* *} \\
(0.04)\end{array}$ & $\begin{array}{c}0.22^{* * *} \\
(0.07)\end{array}$ & & & & \\
\hline Global Macro & & & & & $\begin{array}{l}0.20^{*} \\
(0.11)\end{array}$ & & & & \\
\hline Long/Short Equity Hedge & & & $\begin{array}{c}0.39^{* *} \\
(0.16)\end{array}$ & & & & & & \\
\hline Managed Futures & & & & & & $\begin{array}{c}0.27^{* *} \\
(0.11)\end{array}$ & & & \\
\hline Multi-Strategy & & & & & & & & & $\begin{array}{c}0.29^{* * *} \\
(0.06)\end{array}$ \\
\hline
\end{tabular}

The table provides the estimates of the contagion parameters $\left(c_{k, k^{\prime}}\right)$ for the Poisson model with frailty and contagion. Rows and columns correspond to target and source of contagion, respectively. We set equal to zero the contagion parameters, that are not statistically significant at $5 \%$ in the model with contagion only (Table 4). The free parameters are estimated by the Generalized Method of Moments (GMM) estimator of Section 3.4 iii). Standard errors are provided in parentheses. Stars $*, * *$ and $* * *$ denote significance at the $10 \%, 5 \%$ and $1 \%$ level, respectively. The estimates that are either set equal to zero, or statistically non-significant at $10 \%$ level, are not displayed. 
Table 7: Statistics for diagnostic check of conditional overdispersion and conditional contemporaneous cross-correlation.

\begin{tabular}{|c|c|c|c|c|c|c|c|c|c|}
\hline & $\begin{array}{l}\text { Convertible } \\
\text { Arbitrage }\end{array}$ & $\begin{array}{l}\text { Emerging } \\
\text { Markets }\end{array}$ & $\begin{array}{l}\text { Equity } \\
\text { Market } \\
\text { Neutral }\end{array}$ & $\begin{array}{l}\text { Event } \\
\text { Driven }\end{array}$ & $\begin{array}{c}\text { Fixed } \\
\text { Income } \\
\text { Arbitrage }\end{array}$ & $\begin{array}{l}\text { Global } \\
\text { Macro }\end{array}$ & $\begin{array}{l}\text { Long/Short } \\
\text { Equity Hedge }\end{array}$ & $\begin{array}{l}\text { Managed } \\
\text { Futures }\end{array}$ & $\begin{array}{l}\text { Multi- } \\
\text { Strategy }\end{array}$ \\
\hline Convertible Arbitrage & $2.25^{* *}$ & -0.31 & $2.02^{* *}$ & 1.49 & -0.00 & 0.42 & 1.22 & -1.32 & -0.29 \\
\hline Emerging Markets & & $1.91^{*}$ & 0.39 & 1.40 & 1.19 & 1.37 & $1.97^{* *}$ & -0.09 & 1.61 \\
\hline Equity Market Neutral & & & $2.06^{* *}$ & $1.67^{*}$ & -0.24 & 1.62 & $2.91^{* * *}$ & 1.36 & $1.72^{*}$ \\
\hline Event Driven & & & & $3.82^{* * *}$ & 1.44 & $2.27^{* *}$ & $4.23^{* * *}$ & $1.68^{*}$ & 0.75 \\
\hline Fixed Income Arbitrage & & & & & $1.99^{* *}$ & 0.20 & $2.35^{* *}$ & 1.01 & 0.89 \\
\hline Global Macro & & & & & & $1.79^{*}$ & $2.57^{* *}$ & 0.11 & 0.98 \\
\hline Long/Short Equity Hedge & & & & & & & $4.73^{* *}$ & $2.18^{* *}$ & $2.27^{* *}$ \\
\hline Managed Futures & & & & & & & & $1.83^{*}$ & 1.39 \\
\hline Multi-Strategy & & & & & & & & & $2.60^{* * *}$ \\
\hline
\end{tabular}

The table provides the values of the statistics $\xi_{k l, T}^{*}$, for $k, l=1, \ldots, 9$, defined in equation (3.12). Since the statistic $\xi_{k l, T}^{*}$ is symmetric with respect to the indices $k$ and $l$ of the management styles, we only provide the values on and above the diagonal. Values on the diagonal are used for diagnostic check of conditional overdispersion in any management style. Values outside the diagonal are used for diagnostic check of conditional contemporaneous cross-correlation between any pair of management styles. Under the null hypothesis of no frailty effect, the statistic $\xi_{k l, T}^{*}$ admits a standard normal distribution, for any $k, l$. Stars $*, * *$ and $* * *$ denote significance at the $10 \%, 5 \%$ and $1 \%$ level, respectively. 


\section{Appendix 1: The Autoregressive Gamma process}

In this Appendix we review the main properties of the ARG(1) process used in the paper [see Gourieroux, Jasiak (2006)].

\section{i) The conditional distribution}

The ARG(1) process $\left(F_{t}\right)$ is a Markov process with conditional distribution the noncentral gamma distribution $\gamma\left(\delta, \eta F_{t-1}, \nu\right)$, where $\delta, \delta>0$, is the degree of freedom, $\eta F_{t-1}, \eta>0$, the noncentrality parameter and $\nu$, $\nu>0$, a scale parameter. Its first- and second-order conditional moments are:

$$
E\left(F_{t} \mid F_{t-1}\right)=\delta \nu+\eta \nu F_{t-1}, \quad V\left(F_{t} \mid F_{t-1}\right)=\nu^{2} \delta+2 \eta \nu^{2} F_{t-1}
$$

\section{ii) The state space representation}

The ARG(1) process admits a state space representation, which is especially convenient for simulating the trajectories of the process. To get a simulated value of $F_{t}$ given $F_{t-1}$, we proceed as follows:

a) We draw an intermediate value $Z_{t}^{s}$ in a Poisson distribution $\mathcal{P}\left(\eta F_{t-1}\right)$;

$b)$ Then, $F_{t}$ is drawn in the centered gamma distribution $\gamma\left(\delta+Z_{t}^{s}, 0, \nu\right)$.

\section{iii) Stationarity condition and stationary distribution}

The ARG(1) process is stationary if $\rho=\nu \eta$ is such that $\rho<1$. Then, the stationary distribution is a centered gamma distribution $\gamma\left(\delta, 0, \frac{\nu}{1-\nu \eta}\right)$. In particular, we get the unconditional moments:

$$
E\left(F_{t}\right)=\frac{\nu \delta}{1-\nu \eta}, \quad V\left(F_{t}\right)=\delta\left(\frac{\nu}{1-\nu \eta}\right)^{2} .
$$

From the first equation in (a.1) it is seen that parameter $\rho$ is the first-order autocorrelation of process $\left(F_{t}\right)$.

\section{iv) Normalization and reparameterization}

When the $A R G(1)$ process is used as a latent frailty, the scale of the process can be absorbed in the parameter $\theta$ of the intensity function. Then, the process $\left(F_{t}\right)$ can be normalized to have $E\left(F_{t}\right)=1$. Thus, from the first equation in (a.2), the parameters are such that:

$$
\nu \delta=1-\nu \eta=1-\rho .
$$


It follows that the stationary distribution is $\gamma(\delta, 0,1 / \delta)$, with Laplace transform $E\left[\exp \left(-u F_{t}\right)\right]=(1+u / \delta)^{-\delta}$, for $u>-\delta$. Moreover, the stationary variance is $V\left(F_{t}\right)=1 / \delta$.

\section{Appendix 2: Stationarity and first and second-order moments of the Poisson model with contagion and frailty}

Let us consider the Poisson model with liquidation intensity independent of age, fixed category sizes and ARG frailty dynamics (Assumptions A.3-A.5).

\section{i) Stationarity condition}

Let us consider the joint process $Z_{t}=\left(Y_{t}^{\prime}, F_{t}\right)^{\prime}$, and let $w=\left(u^{\prime}, v\right)^{\prime} \in \mathbb{R}^{K} \times \mathbb{R}$. From a computation similar to equation (2.13), the conditional moment generating function of the Markov process $Z_{t}$ is given by:

$$
E\left[\exp \left(-w^{\prime} Z_{t}\right) \mid Z_{t-1}\right]=\exp \left(-A(w)^{\prime} Z_{t-1}-B(w)\right)
$$

where functions $A(w)$ and $B(w)$ are given by:

$$
A(w)=\left[\sum_{k=1}^{K} c_{k}^{\prime}\left(1-e^{-u_{k}}\right), \alpha\left(v+\sum_{k=1}^{K}\left(1-e^{-u_{k}}\right) b_{k}\right)\right]^{\prime}
$$

and:

$$
B(w)=\sum_{k=1}^{K}\left(1-e^{-u_{k}}\right) a_{k}+\beta\left(v+\sum_{k=1}^{K}\left(1-e^{-u_{k}}\right) b_{k}\right),
$$

and functions $\alpha$ and $\beta$ are given in (2.16). Thus, process $\left(Z_{t}\right)$ is a discrete time affine process. From Proposition 2 in Darolles, Gourieroux, Jasiak (2006), process $\left(Z_{t}\right)$ is strictly stationary if:

$$
\lim _{\tau \rightarrow \infty}\left[\frac{\partial A(0)}{\partial w^{\prime}}\right]^{\tau}=0
$$

Now, by using that $\frac{\partial A(0)}{\partial u_{k}}=\left[c_{k}^{\prime}, b_{k} d \alpha(0) / d u\right]^{\prime}=\left[c_{k}, \rho b_{k}\right]^{\prime}$, for $k=1, \ldots, K$, and $\frac{\partial A(0)}{\partial v}=\left[0^{\prime}, d \alpha(0) / d u\right]^{\prime}=$ $\left[0^{\prime}, \rho\right]^{\prime}$, we get:

$$
\frac{\partial A(0)}{\partial w^{\prime}}=\left(\begin{array}{cc}
C & 0 \\
\rho b^{\prime} & \rho
\end{array}\right) .
$$

Thus, condition (a.4) is satisfied if, and only if, $\rho<1$ and the eigenvalues of matrix $C$ have modulus smaller than 1. 


\section{ii) Moments of order 1}

We have:

$$
E_{t-1}\left(Y_{t}\right)=E_{t-1}\left[E_{t-1}\left(Y_{t} \mid F_{t}\right)\right]=E_{t-1}\left(a+b F_{t}+C Y_{t-1}\right)=a+b E_{t-1}\left(F_{t}\right)+C Y_{t-1},
$$

where $E_{t-1}$ denotes expectation conditional on the past histories of liquidation counts $\underline{Y_{t-1}}$ and factor $\underline{F_{t-1}}$. By taking expectation of both sides of the equation, and using the stationarity of process $\left(Y_{t}\right)$ and the normalization $E\left(F_{t}\right)=1$, we get:

$$
E\left(Y_{t}\right)=a+b+C E\left(Y_{t}\right) \Leftrightarrow E\left(Y_{t}\right)=(I d-C)^{-1}(a+b) .
$$

\section{iii) Moments of order 2}

Let us first consider the covariance between the liquidation counts and the frailty. We have:

$$
\begin{aligned}
E_{t-1}\left(F_{t} Y_{t}\right) & =E_{t-1}\left[E_{t-1}\left(F_{t} Y_{t} \mid F_{t}\right)\right]=E_{t-1}\left[F_{t}\left(a+b F_{t}+C Y_{t-1}\right)\right] \\
& =a E_{t-1}\left(F_{t}\right)+b E_{t-1}\left(F_{t}^{2}\right)+C E_{t-1}\left(F_{t}\right) Y_{t-1} \\
& =a E_{t-1}\left(F_{t}\right)+b E_{t-1}\left(F_{t}^{2}\right)+C\left(1-\rho+\rho F_{t-1}\right) Y_{t-1},
\end{aligned}
$$

from equations (a.1) and (a.3). By taking the expectation of both sides of the equation, we get:

$$
E\left(F_{t} Y_{t}\right)=a+b\left(1+\sigma^{2}\right)+(1-\rho) C(I d-C)^{-1}(a+b)+\rho C E\left(F_{t} Y_{t}\right)
$$

We deduce that:

$$
\begin{aligned}
E\left(F_{t} Y_{t}\right) & =(I d-\rho C)^{-1}\left\{b \sigma^{2}+\left[I d+(1-\rho) C(I d-C)^{-1}\right](a+b)\right\}, \\
& =(I d-\rho C)^{-1} b \sigma^{2}+(I d-C)^{-1}(a+b) .
\end{aligned}
$$

Thus:

$$
\operatorname{Cov}\left(Y_{t}, F_{t}\right)=\sigma^{2}(I d-\rho C)^{-1} b
$$


Let us now consider the variance-covariance matrix of the liquidation counts vector $Y_{t}$. We have:

$$
\begin{aligned}
E_{t-1}\left(Y_{t} Y_{t}^{\prime}\right)= & E_{t-1}\left(E_{t-1}\left[Y_{t} Y_{t}^{\prime} \mid F_{t}\right]\right)=E_{t-1}\left[V_{t-1}\left(Y_{t} \mid F_{t}\right)+E_{t-1}\left(Y_{t} \mid F_{t}\right) E_{t-1}\left(Y_{t} \mid F_{t}\right)^{\prime}\right] \\
= & E_{t-1}\left[\operatorname{diag}\left(a+b F_{t}+C Y_{t-1}\right)\right]+E_{t-1}\left[\left(a+b F_{t}+C Y_{t-1}\right)\left(a+b F_{t}+C Y_{t-1}\right)^{\prime}\right] \\
= & \operatorname{diag}\left[a+b E_{t-1}\left(F_{t}\right)+C Y_{t-1}\right]+b b^{\prime} V_{t-1}\left(F_{t}\right) \\
& +\left[E_{t-1}\left(a+b F_{t}+C Y_{t-1}\right)\right]\left[E_{t-1}\left(a+b F_{t}+C Y_{t-1}\right)\right]^{\prime} \\
= & \operatorname{diag}\left[a+b E_{t-1}\left(F_{t}\right)+C Y_{t-1}\right]+b b^{\prime} V_{t-1}\left(F_{t}\right) \\
& +\left[a+b\left(1-\rho+\rho F_{t-1}\right)+C Y_{t-1}\right]\left[a+b\left(1-\rho+\rho F_{t-1}\right)+C Y_{t-1}\right]^{\prime} .
\end{aligned}
$$

By taking the expectation of both sides, we deduce:

$$
\begin{aligned}
E\left(Y_{t} Y_{t}^{\prime}\right)= & \operatorname{diag}\left[a+b+C(I d-C)^{-1}(a+b)\right]+b b^{\prime} E\left[V_{t-1}\left(F_{t}\right)\right]+V\left[b \rho F_{t-1}+C Y_{t-1}\right] \\
& +E\left[a+b\left(1-\rho+\rho F_{t-1}\right)+C Y_{t-1}\right] E\left[a+b\left(1-\rho+\rho F_{t-1}\right)+C Y_{t-1}\right]^{\prime} \\
= & \operatorname{diag}\left[(I d-C)^{-1}(a+b)\right]+\sigma^{2}\left(1-\rho^{2}\right) b b^{\prime}+V\left[b \rho F_{t-1}+C Y_{t-1}\right]+E\left(Y_{t}\right) E\left(Y_{t}^{\prime}\right),
\end{aligned}
$$

where we used that $E\left[V_{t-1}\left(F_{t}\right)\right]=\sigma^{2}\left(1-\rho^{2}\right)$. Therefore, the variance-covariance matrix of $Y_{t}$ satisfies the recursive equation:

$$
\begin{aligned}
V\left(Y_{t}\right)= & C V\left(Y_{t}\right) C^{\prime}+\operatorname{diag}\left[(I d-C)^{-1}(a+b)\right]+\sigma^{2} b b^{\prime} \\
& +\rho b \operatorname{Cov}\left(F_{t}, Y_{t}\right) C^{\prime}+\rho C \operatorname{Cov}\left(Y_{t}, F_{t}\right) b^{\prime}
\end{aligned}
$$

Equation (2.18) is obtained by substituting the expression (a.6) of $\operatorname{Cov}\left(Y_{t}, F_{t}\right)$.

\section{iv) Autocovariance at order 1}

We have:

$$
\begin{aligned}
\operatorname{Cov}\left(Y_{t}, Y_{t-1}\right) & =\operatorname{Cov}\left[E_{t-1}\left(Y_{t}\right), Y_{t-1}\right]=\operatorname{Cov}\left[E_{t-1}\left(a+b F_{t}+C Y_{t-1}\right), Y_{t-1}\right] \\
& =\operatorname{Cov}\left[a+b\left(1-\rho+\rho F_{t-1}\right)+C Y_{t-1}, Y_{t-1}\right] .
\end{aligned}
$$

Therefore:

$$
\begin{aligned}
\operatorname{Cov}\left(Y_{t}, Y_{t-1}\right) & =\operatorname{Cov}\left(b \rho F_{t-1}+C Y_{t-1}, Y_{t-1}\right)=b \rho \operatorname{Cov}\left(F_{t-1}, Y_{t-1}\right)+C V\left(Y_{t}\right) \\
& =C V\left(Y_{t}\right)+\sigma^{2} \rho b b^{\prime}\left(I d-\rho C^{\prime}\right)^{-1}
\end{aligned}
$$




\section{Appendix 3: Diagnostic checks for the frailty effect}

In this appendix we derive the score for the test of the null hypothesis (3.9) of no frailty effects in the Poisson model. The liquidation count variables $Y_{k, t}$, for $k=1, \ldots, K$, are independent conditionally on $\underline{Y_{t-1}}$ and $\underline{F_{t}}$, with Poisson distribution $\mathcal{P}\left(\gamma_{k, t} \lambda_{k, t}\right)$, where $\lambda_{k, t}=a_{k}+b_{k} F_{t}+c_{k}^{\prime} Y_{t-1}^{*}$. The frailty process $\left(F_{t}\right)$ is a ARG process, normalized with $E\left(F_{t}\right)=1$ and parametrized by $\varphi=(\delta, \rho)^{\prime}$, where parameter $\delta$ is such that $V\left(F_{t}\right)=1 / \delta \equiv \sigma^{2}$ and $\rho$ is the first-order autocorrelation [see Appendix 1 iii) and iv)]. The likelihood function is given by:

$$
L(\theta, \varphi)=\int \ldots \int\left[\prod_{t=1}^{T} \prod_{k=1}^{K} p\left(Y_{k, t} ; \gamma_{k, t} \lambda_{k, t}\right)\right]\left[\prod_{t=1}^{T} g\left(f_{t} \mid f_{t-1} ; \varphi\right)\right] \prod_{t=1}^{T} d f_{t}
$$

where $p(\cdot ; \lambda)$ is the pdf of the Poisson distribution $\mathcal{P}(\lambda)$ with parameter $\lambda>0, g\left(f_{t} \mid f_{t-1} ; \varphi\right)$ is the transition p.d.f. of the ARG process with parameter $\varphi$, and parameter vector $\theta$ is the collection of the vectors $\left(a_{k}, b_{k}, c_{k}^{\prime}\right)^{\prime}$ for all management styles $k$.

We compute the score for the null hypothesis of no frailty effects as the partial derivative of the log-likelihood function w.r.t. parameter $\sigma^{2}$ evaluated at $\sigma^{2}=0$. Let us write the likelihood function as:

$$
L(\theta, \varphi)=\underset{\varphi}{E}\left[\prod_{t=1}^{T} \prod_{k=1}^{K} p\left(Y_{k, t} ; \gamma_{k, t} \lambda_{k, t}^{0}+\gamma_{k, t} b_{k}\left(F_{t}-1\right)\right)\right]
$$

where $\lambda_{k, t}^{0}=a_{k}+b_{k}+c_{k}^{\prime} Y_{t-1}$ and $\underset{\varphi}{E}$ denotes expectation w.r.t the frailty process with parameter $\varphi$. We perform a Taylor series expansion of the function within the expectation around the values $F_{t}=1$ for $t=1, \ldots, T$. We have:

$$
\begin{gathered}
\prod_{t=1}^{T} \prod_{k=1}^{K} p\left(Y_{k, t} ; \gamma_{k, t} \lambda_{k, t}^{0}+\gamma_{k, t} b_{k}\left(F_{t}-1\right)\right)=\exp \left(\sum_{t=1}^{T} \sum_{k=1}^{K} \log p\left(Y_{k, t} ; \gamma_{k, t} \lambda_{k, t}^{0}+\gamma_{k, t} b_{k}\left(F_{t}-1\right)\right)\right) \\
=\prod_{t=1}^{T} \prod_{k=1}^{K} p\left(Y_{k, t} ; \gamma_{k, t} \lambda_{k, t}^{0}\right) \exp \left(\sum_{t=1}^{T} \sum_{k=1}^{K} \frac{\partial \log p\left(Y_{k, t} ; \gamma_{k, t} \lambda_{k, t}^{0}\right)}{\partial \lambda} \gamma_{k, t} b_{k}\left(F_{t}-1\right)\right. \\
\left.+\frac{1}{2} \sum_{t=1}^{T} \sum_{k=1}^{K} \frac{\partial^{2} \log p\left(Y_{k, t} ; \gamma_{k, t} \lambda_{k, t}^{0}\right)}{\partial \lambda^{2}} \gamma_{k, t}^{2} b_{k}^{2}\left(F_{t}-1\right)^{2}+\ldots\right)
\end{gathered}
$$

where the reminder term in the exponent involves powers of $F_{t}-1$ of order larger or equal to 3 . By expanding 
the exponential function, we get:

$$
\begin{aligned}
\prod_{t=1}^{T} \prod_{k=1}^{K} p\left(Y_{k, t} ; \gamma_{k, t} \lambda_{k, t}^{0}+\gamma_{k, t} b_{k}\left(F_{t}-1\right)\right)=\prod_{t=1}^{T} \prod_{k=1}^{K} p\left(Y_{k, t} ; \gamma_{k, t} \lambda_{k, t}^{0}\right)\left(1+\sum_{t=1}^{T} \sum_{k=1}^{K} \frac{\partial \log p\left(Y_{k, t} ; \gamma_{k, t} \lambda_{k, t}^{0}\right)}{\partial \lambda} \gamma_{k, t} b_{k}\left(F_{t}-1\right)\right. \\
\quad+\frac{1}{2} \sum_{t=1}^{T} \sum_{k=1}^{K} \frac{\partial^{2} \log p\left(Y_{k, t} ; \gamma_{k, t} \lambda_{k, t}^{0}\right)}{\partial \lambda^{2}} \gamma_{k, t}^{2} b_{k}^{2}\left(F_{t}-1\right)^{2} \\
\left.\quad+\frac{1}{2} \sum_{t=1}^{T} \sum_{\tau=1}^{T} \sum_{k=1}^{K} \sum_{l=1}^{K} \frac{\partial \log p\left(Y_{k, t} ; \gamma_{k, t} \lambda_{k, t}^{0}\right)}{\partial \lambda} \frac{\partial \log p\left(Y_{l, \tau} ; \gamma_{l, \tau} \lambda_{l, \tau}^{0}\right)}{\partial \lambda} \gamma_{k, t} \gamma_{l, \tau} b_{k} b_{l}\left(F_{t}-1\right)\left(F_{\tau}-1\right)+\ldots\right)
\end{aligned}
$$

We now take the expectation w.r.t. the frailty process, and use that $\underset{\varphi}{E}\left[F_{t}-1\right]=0, \underset{\varphi}{E}\left[\left(F_{t}-1\right)\left(F_{\tau}-1\right)\right]=\rho^{|t-\tau|} \sigma^{2}$, and that higher order central moments of the ARG process involve powers of $\sigma^{2}=1 / \delta$ of order larger or equal to 2. From equation (a.9) we get:

$$
\begin{gathered}
L(\theta, \varphi)=\prod_{t=1}^{T} \prod_{k=1}^{K} p\left(Y_{k, t} ; \gamma_{k, t} \lambda_{k, t}^{0}\right) \\
\left(1+\frac{\sigma^{2}}{2} \sum_{t=1}^{T} \sum_{k=1}^{K} \sum_{l=1}^{K} b_{k} b_{l} \gamma_{k, t} \gamma_{l, t}\left[\frac{\partial^{2} \log p\left(Y_{k, t} ; \gamma_{k, t} \lambda_{k, t}^{0}\right)}{\partial \lambda^{2}} \mathbf{1}\{k=l\}+\frac{\partial \log p\left(Y_{k, t} ; \gamma_{k, t} \lambda_{k, t}^{0}\right)}{\partial \lambda} \frac{\partial \log p\left(Y_{l, t} ; \gamma_{l, t} \lambda_{l, t}^{0}\right)}{\partial \lambda}\right]\right. \\
\left.+\frac{\sigma^{2}}{2} \sum_{t=1}^{T} \sum_{\tau=1, \tau \neq t}^{T} \sum_{k=1}^{K} \sum_{l=1}^{K} b_{k} b_{l} \gamma_{k, t} \gamma_{l, \tau} \rho^{|t-\tau|} \frac{\partial \log p\left(Y_{k, t} ; \gamma_{k, t} \lambda_{k, t}^{0}\right)}{\partial \lambda} \frac{\partial \log p\left(Y_{l, \tau} ; \gamma_{l, \tau} \lambda_{l, \tau}^{0}\right)}{\partial \lambda}+\ldots\right)
\end{gathered}
$$

where the reminder involves terms of order $\sigma^{4}, \sigma^{6}, \ldots$ We deduce the score w.r.t. $\sigma^{2}$ :

$$
\begin{aligned}
& \left.\quad \frac{\partial \log L(\theta, \varphi)}{\partial \sigma^{2}}\right|_{\sigma^{2}=0} \\
& =\frac{1}{2} \sum_{k=1}^{K} \sum_{l=1}^{K} b_{k} b_{l}\left\{\sum_{t=1}^{T} \gamma_{k, t} \gamma_{l, t}\left[\frac{\partial^{2} \log p\left(Y_{k, t} ; \gamma_{k, t} \lambda_{k, t}^{0}\right)}{\partial \lambda^{2}} \mathbf{1}\{k=l\}+\frac{\partial \log p\left(Y_{k, t} ; \gamma_{k, t} \lambda_{k, t}^{0}\right)}{\partial \lambda} \frac{\partial \log p\left(Y_{l, t} ; \gamma_{l, t} \lambda_{l, t}^{0}\right)}{\partial \lambda}\right]\right. \\
& \left.\quad+\sum_{t=1}^{T} \sum_{\tau=1, \tau \neq t}^{T} \rho^{|t-\tau|} \gamma_{k, t} \gamma_{l, \tau} \frac{\partial \log p\left(Y_{k, t} ; \gamma_{k, t} \lambda_{k, t}^{0}\right)}{\partial \lambda} \frac{\partial \log p\left(Y_{l, \tau} ; \gamma_{l, \tau} \lambda_{l, \tau}^{0}\right)}{\partial \lambda}\right\} .
\end{aligned}
$$

By rearranging terms, and using that the density of the Poisson distribution is such that $\log p(y ; \lambda)=y \log \lambda-\lambda$ up to a constant independent of parameter $\lambda$, we get:

$$
\left.\frac{\partial \log L(\theta, \varphi)}{\partial \sigma^{2}}\right|_{\sigma^{2}=0}=\frac{T}{2} \sum_{k=1}^{K} \sum_{l=1}^{K} b_{k} b_{l}\left\{\xi_{k l, T}+\sum_{s=1}^{T-1} \rho^{s}\left[r_{k l, T}(s)+r_{l k, T}(s)\right]\right\}
$$

where the stastistics $\xi_{k l, T}$ and $r_{k l, T}(s)$ are defined in equations (3.10) and (3.11), respectively. 